\title{
Safety and efficacy of enzyme replacement therapy in the nephropathy of Fabry disease
}

\author{
Fernando C Fervenza' \\ Roser Torra ${ }^{2}$ \\ David G Warnock ${ }^{3}$ \\ 'Division of Nephrology \\ and Hypertension, Mayo Clinic \\ College of Medicine, Rochester, MN, \\ USA; '2Department of Nephrology, \\ Fundació Puigvert, Barcelona, Spain; \\ ${ }^{3}$ Division of Nephrology, University \\ of Alabama at Birmingham, \\ Birmingham, AL, USA
}

\begin{abstract}
Kidney involvement with progressive loss of kidney function (Fabry nephropathy) is an important complication of Fabry disease, an X-linked lysosomal storage disorder arising from deficiency of $\alpha$-galactosidase activity. Clinical trials have shown that enzyme replacement therapy (ERT) with recombinant human $\alpha$-galactosidase clears globotriaosylceramide from kidney cells, and can stabilize kidney function in patients with mild to moderate Fabry nephropathy. Recent trials show that patients with more advanced Fabry nephropathy and overt proteinuria do not respond as well to ERT alone, but can benefit from anti-proteinuric therapy given in conjunction with ERT. This review focuses on the use of enzyme replacement therapy with agalsidase-alfa and agalsidase-beta in adults with Fabry nephropathy. The current results are reviewed and evaluated. The issues of dosing of enzyme replacement therapy, the use of adjunctive agents to control urinary protein excretion, and the individual factors that affect disease severity are reviewed.
\end{abstract}

Keywords: agalsidase, enzyme replacement therapy, Fabry nephropathy, anti-proteinuric therapy

\section{Introduction}

Johannes Fabry and William Anderson independently first described this disorder in 1898 (Fabry 2001). They described patients with "angiokeratoma corporis diffusum", the red-purple maculopapular skin lesions that are characteristic of the disease. After these initial cases, other associated symptoms were described and eventually mutations in the alpha-galactosidase A (AGAL) gene were found to be responsible for the disease (Brady et al 1967), which is an X-linked disorder of glycosphingolipid metabolism. This defect results in alpha-galactosidase A deficiency, with progressive accumulation of neutral glycosphingolipids, (predominately globotriaosylceramide; GL-3) in lysosomes, as well as other cellular compartments and the extracellular space (Askari et al 2007). For Fabry disease, the incidence/prevalence ranges from 1 in 40,000 to $1: 117,000$ in the US and Australia to 1:833,000 in northern Portugal, the majority of them Caucasians (Meikle et al 1999). These figures may underestimate the real prevalence of the disease as many patients go undiagnosed due to rarity of this disorder, and phenotypic variation of the clinical features that can be marked, especially in females. Much higher estimates of prevalence (eg, 1 in 4,000) have been obtained with a newborn screening project (Spada et al 2006). Most affected males have little, if any, alpha-galactosidase A activity, and the deposition of GL-3 occurs primarily in vascular endothelial cells as well as epithelial and smooth muscle cells throughout the body. Early clinical manifestations of the disease include angiokeratoma, acroparesthesias, episodic pain "crises", hypohydrosis, and gastrointestinal complaints (Desnick et al 2001).

With time, progressive GL-3 accumulation in the microvasculature and parenchyma leads to microvascular dysfunction, occlusion, and ischemia. Recent reports have described increased oxidative stress (Shen et al 2008), and circulating 
myeloperoxidase in Fabry disease, which appears to be associated with vasculopathic events in male patients (Kaneski et al 2006). The renal, cardiovascular, and cerebrovascular manifestations such as proteinuria, chronic kidney disease and kidney failure, cardiac arrhythmias, hypertrophic cardiomyopathy, and strokes can lead to early death during the fourth and fifth decade of life in affected males (Desnick et al 2001; Branton et al 2002). Kidney involvement in Fabry disease is expressed at an earlier age in hemizygous males than in heterozygous females (Gupta et al 2005; Kobayashi et al 2008; Ortiz et al 2008; Wilcox et al 2008). Ultimately, end-stage renal disease (ESRD) develops in males in the third to fifth decades of life (Desnick et al 2001; Branton et al 2002), although ESRD developing in the second decade has been reported in males (Sheth et al 1983). Females also can progress to ESRD, but generally at a latter age than in males (Wilcox et al 2008).

\section{Clinical presentation and spectrum of Fabry disease}

Patients with Fabry disease presenting only with kidney involvement are rare, and only a few cases have been described (Sawada et al 1996; Nakao et al 2003; Rosenthal et al 2004). Nakao et al screened 514 unselected Japanese male hemodialysis patients, and identified 6 patients with Fabry disease, a prevalence rate of 1.2\% (Nakao et al 2003). Among the cases reported by Nakao et al one had classic Fabry disease that had been overlooked, and 5 patients lacked the "classical" manifestations of angiokeratoma, acroparesthesias, hypohidrosis, and ocular opacities. While one patient had a novel mis-sense mutation (G373D), the others had mutations that were previously described in "classical" cases. Five of the patients (Nakao et al 2003) had left ventricular hypertrophy, and 1 had a normal echocardiogram. It appears that the kidney and heart are frequently involved in Fabry disease. There are overlapping findings between the so-called "cardiac" and "renal" variants (Germain 2001; Nakao et al 2003; Hauser et al 2004; Meehan et al 2004; Fervenza et al 2008); most patients with the cardiac variant also have kidney involvement (Mehta et al 2004). The "cardiac" variant of Fabry disease refers to patients who have some residual alpha-galactosidase A activity, with GL-3 deposition confined to myocytes, and as first described, do not manifest the whole spectrum of symptoms present in classical Fabry disease (von Scheidt et al 1991; Nakao et al 1995). Clinical presentation is usually in the fifth to the eighth decade with left ventricular hypertrophy, mitral insufficiency, and cardiomyopathy and ventricular ectopy (Takenaka et al 2008). It is sobering to note that even with some residual alpha-galactosidase A activity, these patients still develop cardiomyopathy and die with congestive heart failure.

These considerations emphasize the phenotypic variation that can be observed in Fabry disease, even in male patients, and also illustrates the limitations of the term "classical" for describing the presentation of an individual patient. Similarly, use of single organ "variant" descriptions emphasizes major organ involvement at the first assessment, but does not recognize the progressive nature of multi-organ involvement in Fabry disease.

Attempting to correlate the genotype to phenotypic variations is not straightforward. Many female and some male patients have minimal disease manifestations during most of their life. Residual alpha-galactosidase A activity as well as other genetic factors may affect the phenotype. Due to non-random $\mathrm{X}$ inactivation, females appear to have greater phenotypic variation than male patients with the same mutation (Desnick et al 2001; Whybra et al 2001; Gupta et al 2005; Deegan et al 2006; Wilcox et al 2008). Modifying genes may include those involved in glycolipid metabolism, which may increase the availability of substrates for alpha-galactosidase A, thus increasing the disease severity. It has been suggested that a deacylation product of GL-3 may modify disease severity (Aerts et al 2008). Polymorphisms in the 5'-untranslated region could affect the affinity for transcription enhancers and thereby affect AGAL transcription, which could explain altered enzyme activity and even tissue-specific phenotypic expression of Fabry disease in rare patients for whom coding region or splice-site mutations cannot be readily identified (Oliveira et al 2008).

\section{Kidney involvement in Fabry disease}

Since Fabry disease is a potentially treatable condition it is imperative to consider it in the differential diagnosis of any patient presenting with progressive chronic kidney disease (CKD) and proteinuria, especially if the blood pressure is not elevated and there is a family history of kidney disease. While Fabry disease may not lead the differential diagnosis list, the clinical indications for kidney biopsy are clearly fulfilled, and the results should readily lead to the correct diagnosis, even if it was not suspected before the procedure. The typical manifestations of Fabry nephropathy on biopsy are well described and readily appreciated, so the diagnosis should be readily discerned (Gubler et al 1978; Sessa et al 2002; Fervenza et al 2008). 
Two patient registries are now available (Fabry Registry and Fabry Outcome Survey), which contain detailed descriptions of individual patient status. Baseline assessments of age and estimated glomerular filtration rate (eGFR) for 585 adult male and 677 adult female patients from the Fabry Registry have been published (Ortiz et al 2008). Thirteen percent of adult females, and $28 \%$ of adult males had eGFR $<60 \mathrm{~mL} / \mathrm{min} / 1.73 \mathrm{~m}^{2}$ at their baseline evaluations before starting enzyme replacement therapy (ERT). Figure 1 (Ortiz et al 2008) illustrates the magnitude of proteinuria at the time of baseline evaluation for 300 male (top panel) and
306 female (bottom panel) members of the Fabry Registry cohort for whom these data were available. The horizontal lines represent the median level of proteinuria, and the vertical lines represent the median eGFR. Overt proteinuria is present at the first evaluation in the majority of patients, throughout the entire range of eGFR.

\section{Enzyme replacement therapy (ERT)}

Until recently, recognition of Fabry disease did not affect the patient's prognosis, since no treatment was available. However, the availability of ERT with recombinant

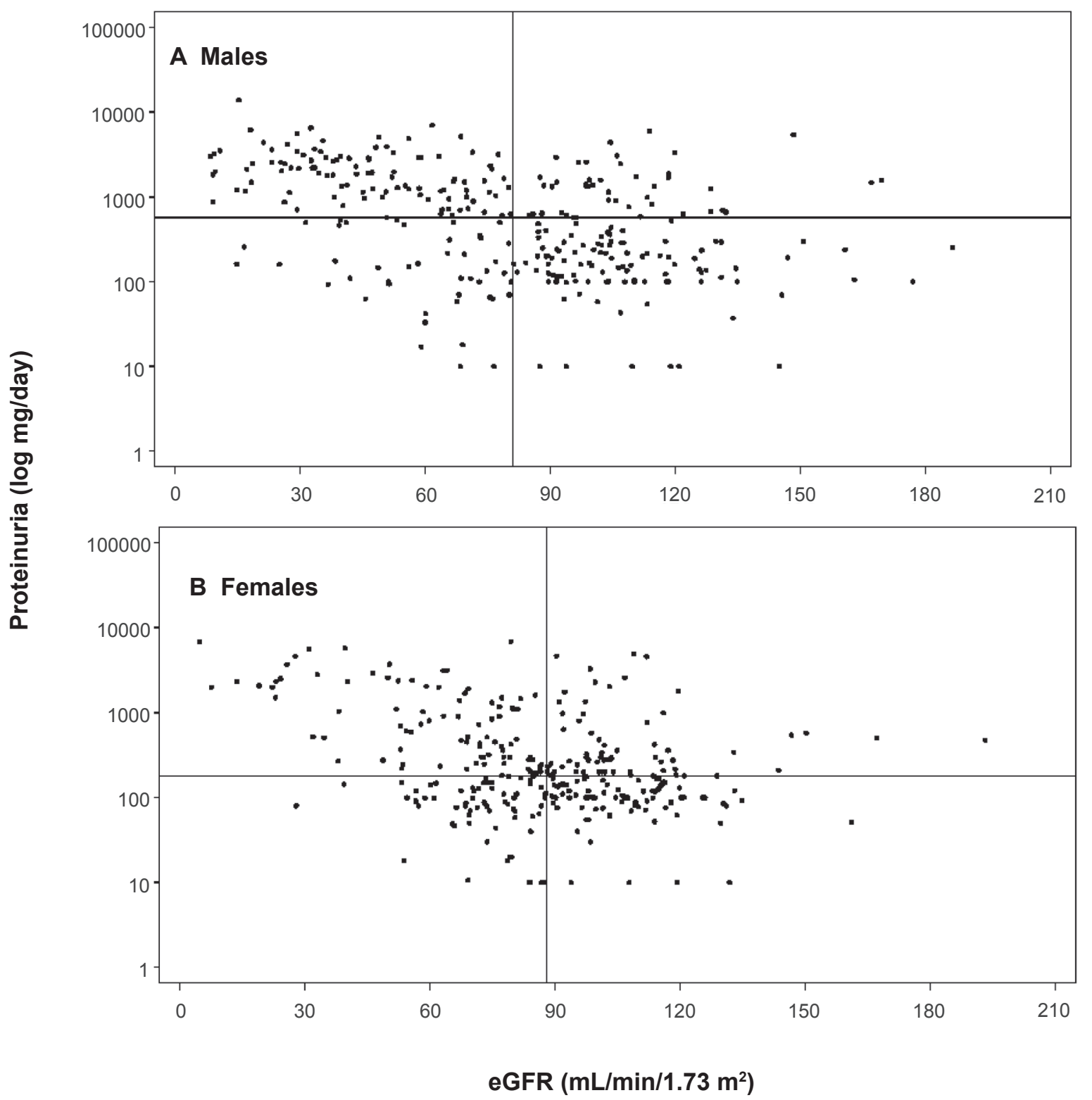

Figure I Distribution of proteinuria and eGFR.The median values for eGFR are shown as vertical lines, and the median 24-h urine protein is shown as horizontal lines in both panels. A) Males, $\mathrm{n}=300$, median eGFR $=81.0 \mathrm{~mL} / \mathrm{min} / 1.73 \mathrm{~m}^{2}$, and median proteinuria $=572 \mathrm{mg} / 24 \mathrm{~h}$. B) Females, $\mathrm{n}=306, \mathrm{median}$ eGFR $=88.0 \mathrm{~mL} / \mathrm{min} / \mathrm{l} .73 \mathrm{~m}{ }^{2}$, and $\mathrm{median}$ proteinuria $=$ $180 \mathrm{mg} / 24 \mathrm{hr}$. Data from the Fabry Registry reproduced with permission from Ortiz A, Oliveira JP,Waldek S, et al 2008. Nephropathy in males and females with Fabry disease: cross-sectional description of patients before treatment with enzyme replacement therapy. Nephrol Dial Transplant, 23:1600-7. Copyright @ 2008 Oxford University Press. 
alpha-galactosidase A offers the promise of altering the natural history of this rare form of proteinuric CKD (Schiffmann 2007; Warnock 2007).

Currently, there are two forms of ERT available for the treatment of Fabry disease: (1) Replagal ${ }^{\circledR}$ (agalsidase-alfa; Shire Human Genetic Therapies, Inc., Cambridge, MA) and (2) Fabrazyme ${ }^{\circledR}$ (agalsidase-beta; Genzyme Corporation, Inc., Cambridge, MA). With the exception of the structures of the oligosaccharide side chains, the primary amino acid sequences of these products are the same (Blom et al 2003). The approved doses of agalsidase-alfa and agalsidase-beta are $0.2 \mathrm{mg} / \mathrm{kg}$ and $1.0 \mathrm{mg} / \mathrm{kg}$, given intravenously every 2 weeks, respectively. Agalsidase-beta has been approved for the treatment of Fabry disease in the US, while both agents are available for clinical use in other countries (Desnick 2004). The overall experience with ERT has been very encouraging (Barbey et al 2008). Figure 2 illustrates the effect of ERT in a male Fabry patient with moderately severe nephropathy in whom proteinuria was controlled with irbesartan (De Schoenmakere et al 2003); the rate of loss of kidney function was reduced by $67 \%$. Earlier reports with agalsidase-alfa given at $0.2 \mathrm{mg} / \mathrm{kg}$ every other week to male Fabry patients with relatively mild disease were also encouraging (Barbey et al 2008).

\section{Placebo-controlled trials of ERT}

Agalsidase-alfa phase III trial

The first double-blind, placebo controlled trial with agalsidase-alfa at $0.2 \mathrm{mg} / \mathrm{kg}$ given every 2 weeks for 24 weeks was carried out in 24 "classically affected" adult male Fabry patients, with average age of 34.2 years, and creatinine clearance of $96 \mathrm{~mL} / \mathrm{min}$ (Schiffmann et al 2001). The primary outcome measure, reduction in neuropathic pain "at its worst, without pain medications", was improved in the 13 active-treatment patients compared to the placebo-treated controls. Secondary outcome measures included stabilization of creatinine and inulin clearances in the active-treatment group, while the placebo-controlled group of 11 patients had approximately 18\% decreases in their kidney function. Baseline urine protein excretion exceeded $1 \mathrm{~g} /$ day in 5 of the active-treatment group and 3 of the placebo-treated group. Mesangial widening decreased by $12.5 \%$ in kidney biopsies evaluated before and after 24 weeks of agalsidase-alfa therapy, and increased by $16.5 \%$ in the placebo-treated patients.

\section{Proteinuria}

\section{$0.4-0.6$ grams}

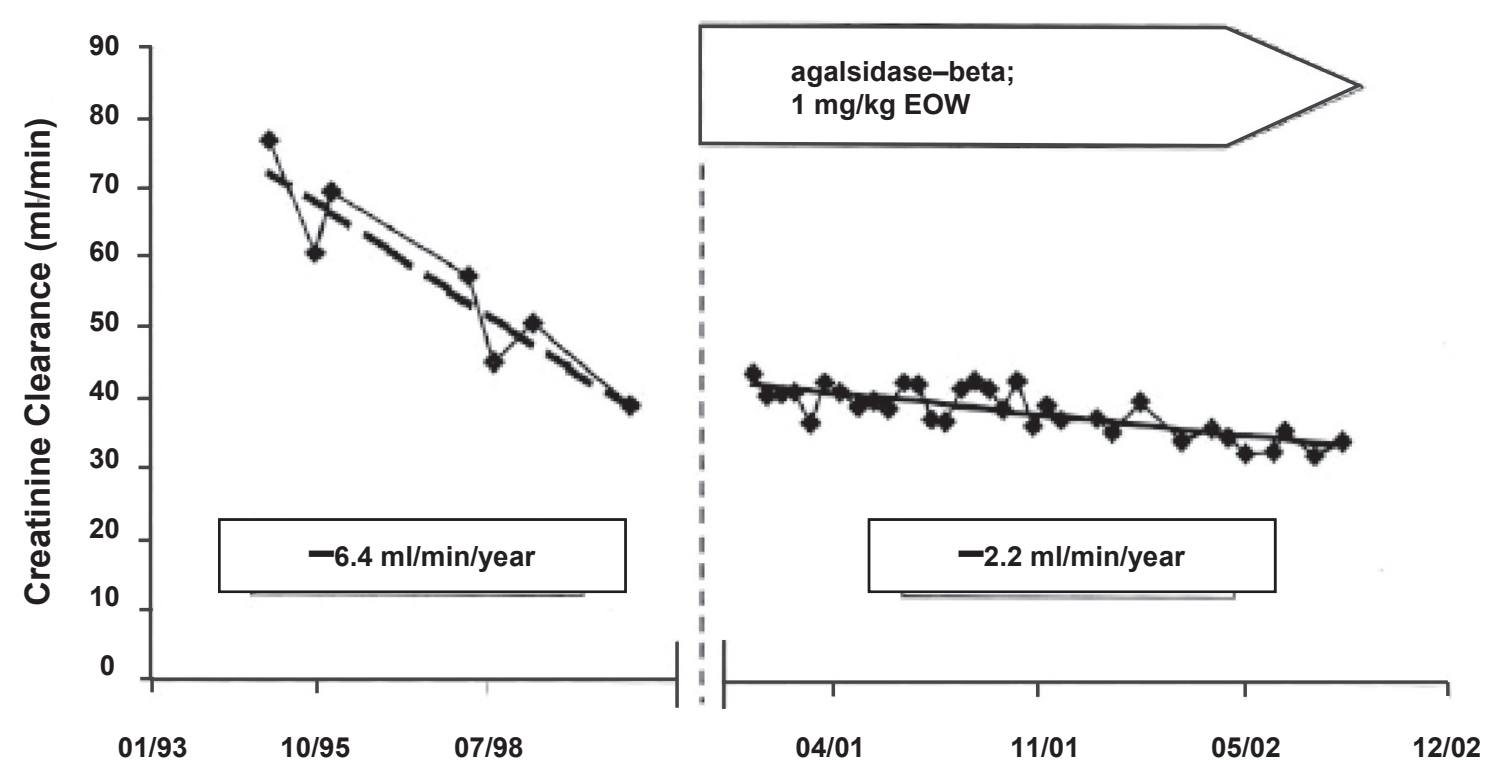

Date (month/year)

Figure 2 Effects of agalsidase-beta on rate of loss of kidney function in a 36-year-old male with Fabry nephropathy. Creatinine clearance was measured at the indicated points, and enzyme replacement therapy ( $\mathrm{I} \mathrm{mg} / \mathrm{kg}$ every 2 weeks) was started in early $200 \mathrm{I}$. During the entire follow-up period, there were no major changes in blood pressure or proteinuria (0.4-0.6 g). The patient was started on $5 \mathrm{mg}$ lisinopril in 1994. Because of persistent cough, irbesartan at $150 \mathrm{mg} /$ day was substituted in for lisinopril in December 200I. Reproduced with permission from De Schoenmakere G, Chauveau D, Grunfeld JP. 2003. Enzyme replacement therapy in Anderson-Fabry's disease: beneficial clinical effect on vital organ function. Nephrol Dial Transplant, 18:33-5. Copyright @ 2003 Oxford University Press. 
Surprisingly, there was a significant increase in the segmental sclerosis scores for the active-treatment group after 24 weeks. This effect may have reflected the presence of overt proteinuria in 5 of the active-treated patients. There was no effect on proteinuria observed with 24 weeks of agalsidasealfa therapy.

Most (8/14) of the active-treatment patients had mild infusion-related reactions, and 12/14 developed IgG antibodies to agalsidase. Plasma GL-3 decreased in the active-treatment group, and was unchanged in the placebo-treated group. Urinary GL-3 decreased by $29 \%$ in the active-treatment group, and increased by $15.4 \%$ in the placebo-treated patients.

\section{Agalsidase-beta phase III trial}

Results of a multi-center double blind phase III trial in which 58 patients (average age 30.2 years, 56 males) were randomized to agalsidase-beta or placebo for 20 weeks followed by 6 months open-label treatment (Eng et al 2001). The primary outcome measure was reduction in GL-3 accumulation scores to zero in renal interstitial capillaries. Twenty out of 29 patients $(69 \%)$ in the agalsidase-beta treatment group, and none of the placebo-treated patients achieved complete GL-3 clearance from their renal interstitial capillaries $(p<0.001)$. Six of the other 9 active treatment group patients showed substantial but not complete improvement in their GL-3 accumulation scores.

Secondary outcome measures included reduction of microvascular endothelial deposits in heart, kidney and skin, change in baseline urinary GL-3 excretion and kidney GL-3 content, and reduction in pain scores. The overall composite score for microvascular endothelial GL-3 accumulation decreased from $4.9 \pm 1.5$ at baseline to $0.7 \pm 0.8$ at week 20 ( $85.7 \%$ decrease). The baseline GFR measured with inulin clearance was $83 \mathrm{ml} / \mathrm{min}$ in the active-treatment group, and $96.6 \mathrm{~mL} / \mathrm{min}$ in the placebo-treatment group at baseline. Kidney function did not significantly change in either group at the end of the 24-week double-blind study ( $p<0.19$ ), or after 6 months of open label treatment ( $p<0.81)$. Fifty nine percent of the active-treated group had mild infusion related reactions, and $88 \%$ had $\mathrm{IgG}$ seroconversion. Plasma GL-3 decreased to normal levels by week 14 in the active-treated group, and did not change in the placebo-treated group. Urinary GL3 decreased by $34.1 \%$ in the active-treatment group, and by $6.2 \%$ in the placebo-treatment group.

\section{Agalsidase-beta phase IV trial}

Results of a multi-center double-blind phase IV trial in which 82 adult patients with initial GFR values $<80 \mathrm{~mL} / \mathrm{min} / 1.73 \mathrm{~m}^{2}$ who were randomized $(2: 1)$ to treatment with agalsidase-beta at $1 \mathrm{mg} / \mathrm{kg}$ every 2 weeks or placebo have been published (Banikazemi et al 2007). Seventy-two patients were males, and the average age was 45.9 years. The median time in treatment was 18.5 months, and individual patients were treated for as long as 35 months.

The composite primary endpoint was the first clinical event: kidney $-33 \%$ increase in creatinine on 2 successive measures or reaching ESRD; cardiac events; central nervous system (CNS) events; or death. The expected event rate was $40 \%$ over 2 years for the placebo group, and $10 \%$ for the active-treatment patients. The study ended when approximately one third of the patients had experienced a total of 27 primary clinical events, 17 (63\%) of which were accounted by sustained $33 \%$ increases in serum creatinine.

Primary endpoint analysis was conducted on the intention-to-treat cohort, and the secondary analyses, in a pre-specified per-protocol population. Two deaths, 1 for each group, occurred after a primary endpoint (cardiac arrest, and cardiac arrest after stroke), and death of 1 patient (due to pulmonary embolism) in the agalsidase-beta group was the primary endpoint. Thirteen of 31 patients in the placebo group (41.9\%) had primary outcome events (7 renal events, 4 cardiac, 2 CNS, 0 death), and 14 of the 51 agalsidase-beta patients $(27.4 \%)$ had primary outcome events (10 renal events, 3 cardiac, 0 CNS, 1 death from pulmonary embolus).

There was an imbalance in the baseline proteinuria, with patients allocated to the ERT arm having significantly greater baseline proteinuria than those in the placebo arm, a fact that complicates the interpretation of the outcome of the study (Schiffmann 2007). The baseline eGFR was 53.0 17.7 (SD) in the agalsidase-beta group and $52.4 \pm 17.7$ (SD) in the placebo group. The baseline urine protein/creatinine ratio was $1.5= \pm 1.5$ (SD) in the agalsidase-beta group, and $1.1 \pm 1.4(\mathrm{SD})$ in the placebo group. After adjustment for baseline proteinuria, the intention-to-treat analysis showed that ERT was associated with a 53\% risk reduction in the primary event rate, although due to the small number of patients in the trial the results did not reach statistically significance $(\mathrm{p}=0.058)$. Secondary analysis showed that the benefit of ERT was greater in those patients with GFR values $>55 \mathrm{~mL} / \mathrm{min}$ per $1.73 \mathrm{~m}^{2}$, and those patients with GFR values $<55 \mathrm{~mL} / \mathrm{min}$ per $1.73 \mathrm{~m}^{2}$ did not appear to benefit from ERT (Banikazemi et al 2007).

Most of the treatment-related events were mild or moderate infusion-associated reactions (rigors and fever) that occurred in $55 \%$ of patients in the agalsidase-beta group and $23 \%$ of the placebo-treated group, and were more common 
during the first 6 months of treatment. One patient in the agalsidase-beta group experienced severe hypotension and had a positive serum IgE test result. Forty-three patients (68\%) developed IgG antibodies against recombinant agalsidase-beta. Plasma GL-3 levels were reduced to normal by 6 months in the active-treatment group, and were maintained at these levels throughout the study. Urinary GL-3 levels were not assessed.

\section{Open label studies of ERT dose and frequency of dosing}

The effect of dosing interval with agalsidase-alfa has been examined (Schiffmann et al 2007); a group of adult males patients who had progressive decline in eGFR despite 2 to 4 years of $0.2 \mathrm{mg} / \mathrm{kg}$ agalsidase-alpha therapy every other week, were $0.2 \mathrm{mg} / \mathrm{kg}$ agalsidase-alfa every week. (It should be noted that this is an "off-label" use of agalsidase-alfa.) Before switching to weekly dosing, the mean decline in eGFR was $-8.0 \pm 2.8(\mathrm{SD}) \mathrm{mL} / \mathrm{min} / 1.73 \mathrm{~m}^{2} /$ year. Four patients with low baseline proteinuria (average $=222.8 \pm 60[\mathrm{SD}] \mathrm{mg} /$ day) progressed on agalsidase-alfa given every other week at a rate of $-6.6 \pm 2.1 \mathrm{~mL} / \mathrm{min} / 1.73 \mathrm{~m}^{2}$ per year. After switching to weekly dosing of agalsidase-alfa, these 4 patients had an improvement in their kidney function (Figure 3 ). The remaining patients with higher baseline proteinuria levels had some improvement in the rate of decline of their kidney function, but still progressed at a rate of $-5.5 \pm 4.2$ (SD) $\mathrm{mL} / \mathrm{min} / 1.73 \mathrm{~m}^{2}$ per year on $0.2 \mathrm{mg} / \mathrm{kg}$ agalsidase-alfa given on a weekly basis (Figure 3 ).

A similar experience with increasing the dose of agalsidase-alfa from $0.2 \mathrm{mg} / \mathrm{kg}$ every other week to $0.4 \mathrm{mg} / \mathrm{kg}$ every other week in an off-label, compassionate-use basis has been described (Torra et al 2008). This patient had baseline proteinuria that ranged from 1 to $3 \mathrm{~g}$ /day, and developed moderately severe Fabry nephropathy with a progression rate of $-7.7 \pm 1.2 \mathrm{~mL} / \mathrm{min} / 1.73 \mathrm{~m}^{2} /$ year before the institution of ERT (Figure 4). No significant effect on kidney function was seen with agalsidase-alfa given at $0.2 \mathrm{mg} / \mathrm{kg}$, and when the dose was increased (off-label, compassionate use) to $0.4 \mathrm{mg} / \mathrm{kg}$ every other week, the progression rate slowed to $-3.7 \pm 0.6 \mathrm{~mL} / \mathrm{min} / 1.73 \mathrm{~m}^{2} /$ year. While consistent with a dose-effect of ERT on progression, this effect was also confounded by overt proteinuria of $2.7 \pm 0.5 \mathrm{~g} /$ day during the period of agalsidase-alfa administration at $0.2 \mathrm{mg} / \mathrm{kg}$ every 2 weeks and reduced proteinuria associated with ACEI therapy during the later phase.

Another case report (Warnock 2005) reached a similar conclusion with respect to the dosing of ERT in moderately

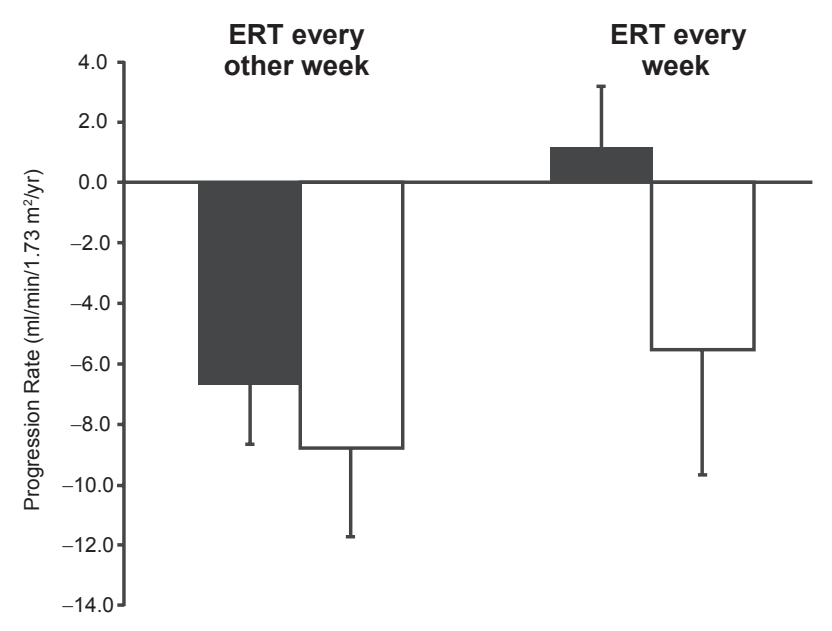

Figure 3 Annualized change in estimated glomerular filtration rate $(\mathrm{mL} / \mathrm{min} / \mathrm{l} .73$ $\mathrm{m}^{2} /$ year $\pm \mathrm{SD}$ ) in patients treated with agalsidase-alfa at $0.2 \mathrm{mg} / \mathrm{kg}$ every 2 weeks (left bars) followed by weekly treatment (right bars). Patients are stratified by baseline proteinuria: $<0.3 \mathrm{~g} /$ day, black bars; $>$ I g/day, open bars. Adapted with permission from Schiffmann R, Askari H, Timmons M, et al 2007. Weekly enzyme replacement therapy may slow decline of renal function in Fabry patients who are on long-term biweekly dosing. J Am Soc Nephrol, 18:1576-83. Copyright (c) 2007 American Society of Nephrology.

severe Fabry nephropathy. This patient was initially treated with agalsidase-alfa; despite anti-proteinuric therapy with enalapril and losartan to control proteinuria before ERT was started, and at an average $0.83 \pm 0.54 \mathrm{~g} /$ day during the administration of ERT at $0.2 \mathrm{mg} / \mathrm{kg}$ every other week, there was rapid progression at $-13.7 \pm 1.5 \mathrm{~mL} / \mathrm{min} / 1.73 \mathrm{~m}^{2} /$ year during the 16 months of treatment with agalsidase-alfa (Figure 5). This patient was then switched to agalsidase-beta in May 2003, and was the first patient to receive commercially available ERT in the US (Warnock 2005). This patient progressed rapidly on agalsidase-alfa $(0.2 \mathrm{mg} / \mathrm{kg}$ every other week) despite control of his proteinuria with ACEI/ARB therapy before ERT was instituted (Figure 5), suggesting that control of proteinuria is necessary but not sufficient for controlling progressive loss of eGFR in moderately severe Fabry nephropathy, and should not be considered as a treatment alternative to the initiation of ERT in Fabry nephropathy.

An open-label extension study of the phase IV study with agalsidase-beta has been completed, but the results have not yet been published. In post-hoc analysis of the original phase IV report, an apparent benefit of ERT with agalsidase-beta at $1 \mathrm{mg} / \mathrm{kg}$ every 2 weeks was observed in patients with baseline eGFR $>55 \mathrm{ml} / \mathrm{min} / 1.73 \mathrm{~m}^{2}$; there was a $75 \%$ reduction in the hazard ratio compared to placebo for reaching the primary endpoint (Banikazemi et al 2007). It is important to know if the renal protective effect of ERT was maintained throughout the 18-month follow-up period of 


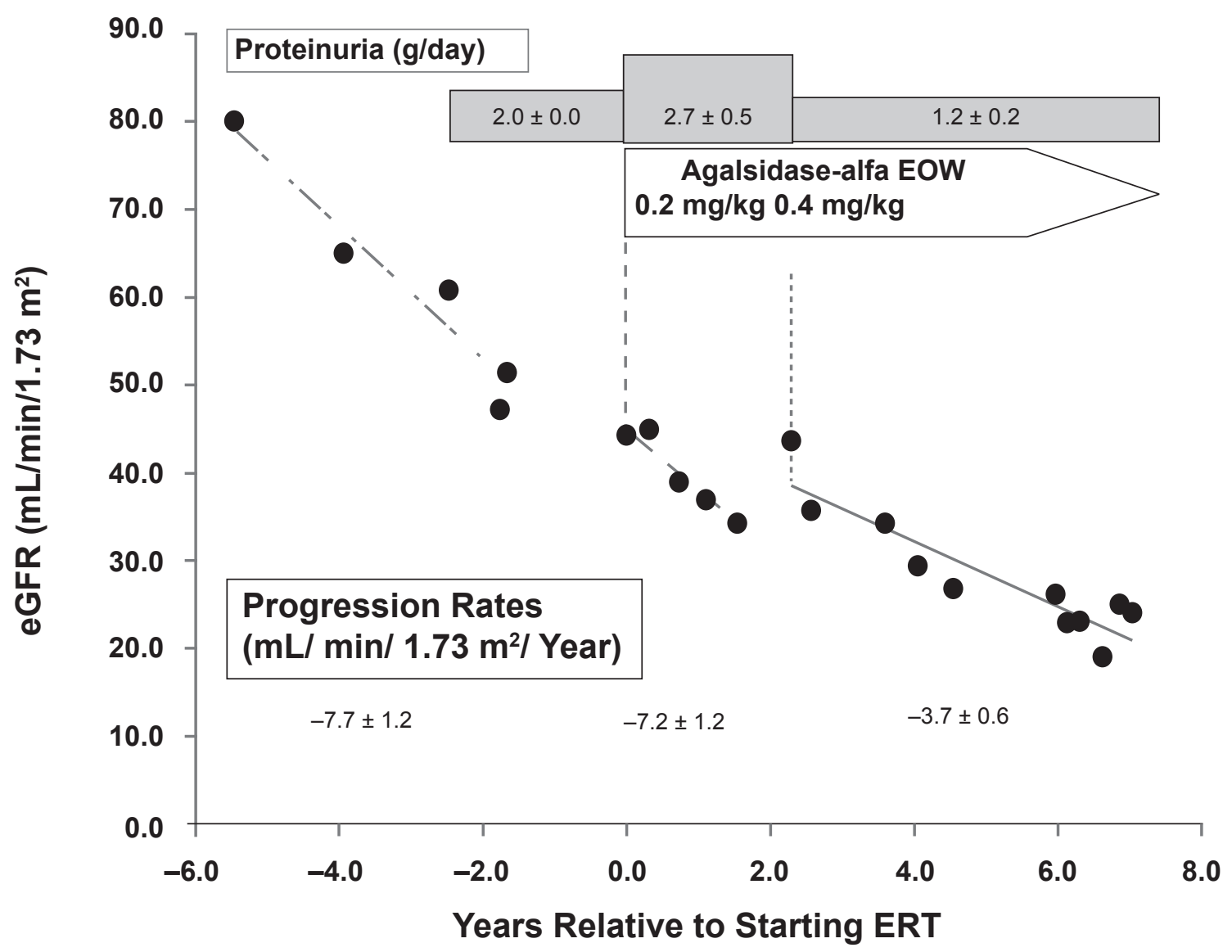

Figure 4 Annualized progression rate of change in estimated glomerular filtration rate $\left(\mathrm{mL} / \mathrm{min} / \mathrm{I} .73 \mathrm{~m}^{2} /\right.$ year $\left.\pm \mathrm{SD}\right)$ for a male patient before ERT, and during treatment with agalsidase-alfa at $0.2 \mathrm{mg} / \mathrm{kg}$ every 2 weeks, followed by treatment with agalsidase-alfa at $0.4 \mathrm{mg} / \mathrm{kg}$ every 2 weeks. The progression slope was reduced in half with the higher dose of ERT. The urine protein excretion averaged I.98 \pm 0.03 (SD) g/24 h before ERT, $2.72 \pm 0.52$ (SD) g/24 h during ERT at $0.2 \mathrm{mg} / \mathrm{kg}$ every other week, and I. $17 \pm 0.22$ (SD) $\mathrm{g} / 24 \mathrm{~h}$ during ERT at $0.4 \mathrm{mg} / \mathrm{kg}$ every other week along with enalapril at $5 \mathrm{mg} 3$ times per day.Adapted with permission Torra R,Algaba F,Ars E, et al 2008. Preservation of renal function in a patient with Fabry nephropathy on enzyme replacement therapy. Clin Nephrol, 69:445-9. Copyright (C) 2008 Dustri-Verlag.

the phase IV extension study. Similar long-term follow up studies of the patients enrolled in the agalsidase-alfa studies would also be of great interest.

\section{Endothelial GL-3 clearance and the effects of ERT}

Randomized, placebo-controlled trials and long-term, open-label extension studies of both products have consistently demonstrated that ERT reduces GL-3 levels in plasma and urine as well as glycosphingolipids accumulation in capillary endothelial cells, renal glomerular cells, and tubular epithelial cells (Schiffmann et al 2001; Thurberg et al 2002), although the extent of clearance may be greater with the larger doses of ERT. A detailed examination of the effects of ERT on GL-3 deposits in various kidney cell types has been described by Thurburg et al (Thurberg et al 2002).

An example of severe GL-3 deposits in the glomerular capillary endothelial cells of a patient with severe Fabry nephropathy (baseline eGFR $=25 \mathrm{ml} / \mathrm{min} / 1.73 \mathrm{~m}^{2}$; baseline proteinuria $=3.4 \mathrm{~g} /$ day in September, 2006), and the complete resolution of the endothelial deposits after 14 months of ERT with agalsidase-beta at $1 \mathrm{mg} / \mathrm{kg}$ every 2 weeks is shown in Figure 6. The glomerular epithelial cells still contain lamellar inclusions; these are minimally cleared of GL-3 even after extended course of ERT (Germain et al 2007). An autopsy report confirms the complete clearance of vascular endothelial GL-3 in a male treated with agalsidasebeta for 2 years (Schiffmann et al 2005). Also notable in Figure 6D is effacement of the epithelial foot processes, as has been recently described (Valbuena et al 2008), while after 15 months of ERT, and reduction of proteinuria with ACEI/ARB therapy, the foot processes appear to have been restored.

Is there a dose-dependent effect of ERT on capillary endothelial GL-3 clearance? Systematic studies with doses less than $1 \mathrm{mg} / \mathrm{kg}$ given every 2 weeks have not been reported in detail. Schiffmann et al reported a "decrease in glycolipid inclusions within vascular endothelium" in a group of male 


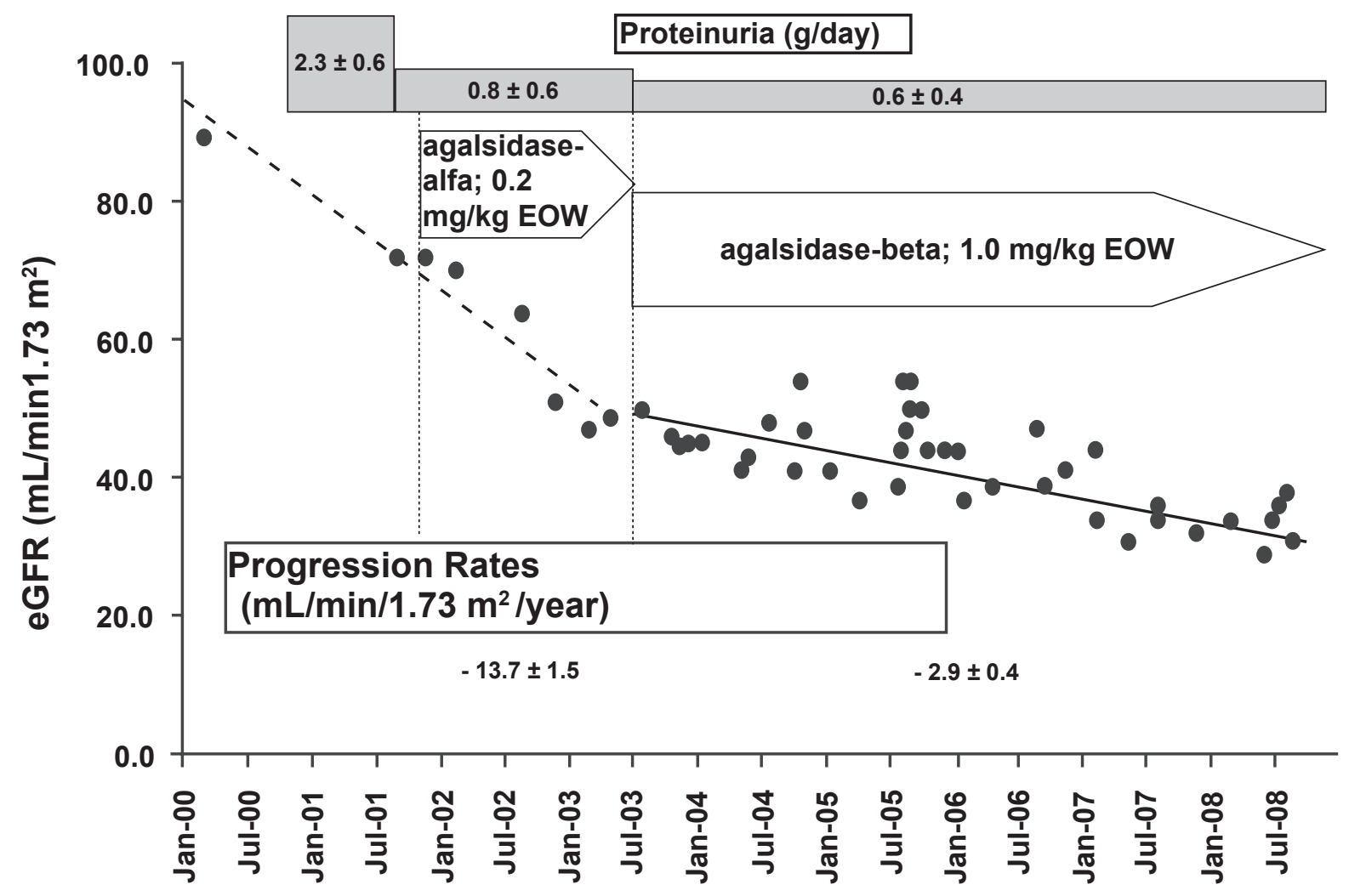

Date (month/year)

Figure 5 Annualized progression rate of change in estimated glomerular filtration rate $\left(\mathrm{mL} / \mathrm{min} / 1.73 \mathrm{~m}^{2} /\right.$ year $\left.\pm \mathrm{SD}\right)$ for a male patient during treatment with agalsidase-alfa at $0.2 \mathrm{mg} / \mathrm{kg}$ every 2 weeks, followed by treatment with agalsidase-beta at $1.0 \mathrm{mg} / \mathrm{kg}$ every 2 weeks. The progression slope was reduced by $77 \%$ with the higher dose of ERT.The average baseline proteinuria was $2.27 \pm 0.0 .57$ (SD) g/day before any treatment, and with added enalapril (10 mg) and losartan ( $50 \mathrm{mg})$, was reduced to before any ERT to 1.03 $\mathrm{g} /$ day, and was maintained at an average of $0.83 \pm 0.54(\mathrm{SD}) \mathrm{g} /$ day during agalsidase-alfa treatment, and $0.56 \pm 0.24$ (SD) g/day during agalsidase-beta treatment. Adapted with permission from Warnock DG. 2005. Fabry disease: diagnosis and management, with emphasis on the renal manifestations. Curr Opin Nephrol Hypertens, 14:87-95. Copyright () 2005 Lippincott Williams \& Wilkins.

patients treated with $0.2 \mathrm{mg} / \mathrm{kg}$ agalsidase-alfa every 2 weeks for 24 weeks (Schiffmann et al 2001), but the extent of the reduction was not specified, and complete clearing of GL-3 deposits was not described (Schiffmann et al 2001). Several patients have been reported in which the kidney biopsies show persisting glomerular capillary endothelial GL-3 deposits despite ERT with $0.2 \mathrm{mg} / \mathrm{kg}$ given every 2 weeks. Figure 7 shows 3 such cases, one treated with $0.2 \mathrm{mg} / \mathrm{kg}$ given every 2 weeks for 16 months, another for 24 months, and another for 60 months. The red circles localize what appear to be persisting, dense endothelial deposits of GL-3. In contrast, the capillary endothelial cells seen in Figure 7 do not have such deposits after 14 months of agalsidase-beta therapy given at $1.0 \mathrm{mg} / \mathrm{kg}$ every 2 weeks.

Fundamental questions have to be answered before microvascular GL-3 clearance can be considered as a valid surrogate outcome marker for the treatment effects of ERT in Fabry disease. More information is needed about the relationship between the total accumulation of GL-3 and the burden of disease in the individual patient. Measurements of tissue levels of GL-3 are invasive, so more readily accessible samples, such as urinary GL-3 excretion are obviously desirable. There are some preliminary data available in the description of the natural history of male Fabry patients (Branton et al 2002) that support the interest in using GL-3 measurements as surrogate markers for the underlying disease pathogenesis in Fabry disease. As shown in Figure 8, there is a relationship between the amount of GL-3 in the kidney, as assessed by determinations of GL- 3 content in kidney biopsy specimens, and the severity of glomerular damage (Figure 8A), as well as the urinary excretion of GL-3 (Figure 8B). Males have higher plasma and urinary levels of GL-3 than females, and the plasma and urinary GL-3 levels correlate with each other, however, there is not correlation between elevated GL-3 levels and clinical symptoms (Vedder et al 2007). The validation of microvascular 

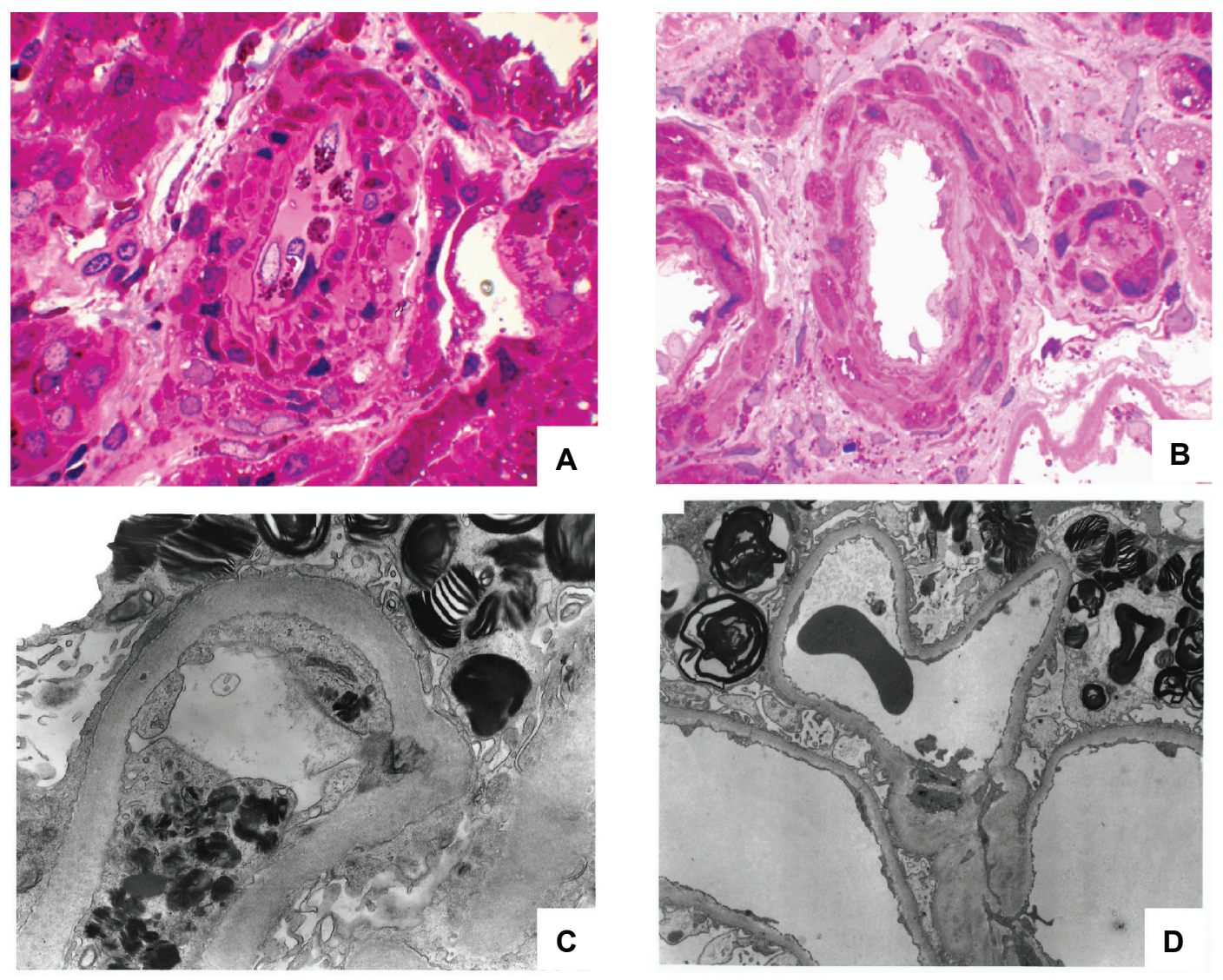

November 2004

January 2006

Figure 6 Effects of agalsidase-beta on arteriolar intimal and medial, and glomerular capillary endothelial GL-3 deposits. The patient was a 33-year-old male who was diagnosed with Fabry disease on the basis of the kidney biopsy findings.At the time of the initial biopsy, his estimated glomerular filtration rate was $25 \mathrm{~mL} / \mathrm{min} / 1.73 \mathrm{~m} \mathrm{~m}^{2}$, and his urine protein excretion was $3.3 \mathrm{~g} / 24 \mathrm{~h}$. His proteinuria was controlled to an average of $0.66 \pm 0.44$ (SD) g/24 h with $20 \mathrm{mg}$ enalapril and $150 \mathrm{mg}$ irbesartan, and agalsidase-beta treatment was started at $1.0 \mathrm{mg} / \mathrm{kg}$ every other week. The kidney biopsy was repeated after 15 months of ERT therapy. A) Renal cortical arteriole, before starting ERT (Masson-trichrome stain). B) Renal cortical arteriole, 15-months after starting ERT (Masson-trichrome stain); note clearing of endothelial and intimal GL-3 deposits. C) Glomerular capillary loop, before starting ERT (electron micrograph); note the dense endothelial deposits with substantial obliteration of the capillary lumen and the "Zebra bodies" in the podocytes. D) Glomerular capillary loop, I5 months after starting ERT (electron micrograph); note that the endothelial deposits have cleared, but "Zebra bodies" persist in the podocytes. The magnification is the same for $\mathbf{A}$ and $\mathbf{B}$, but $\mathbf{C}$ and $\mathbf{D}$ have different magnification factors. For point of reference, the basement membrane thickness is the same for the electron micrographs before and after ERT. Courtesy of William Cook, MD PhD Department of Pathology, University of Alabama at Birmingham.
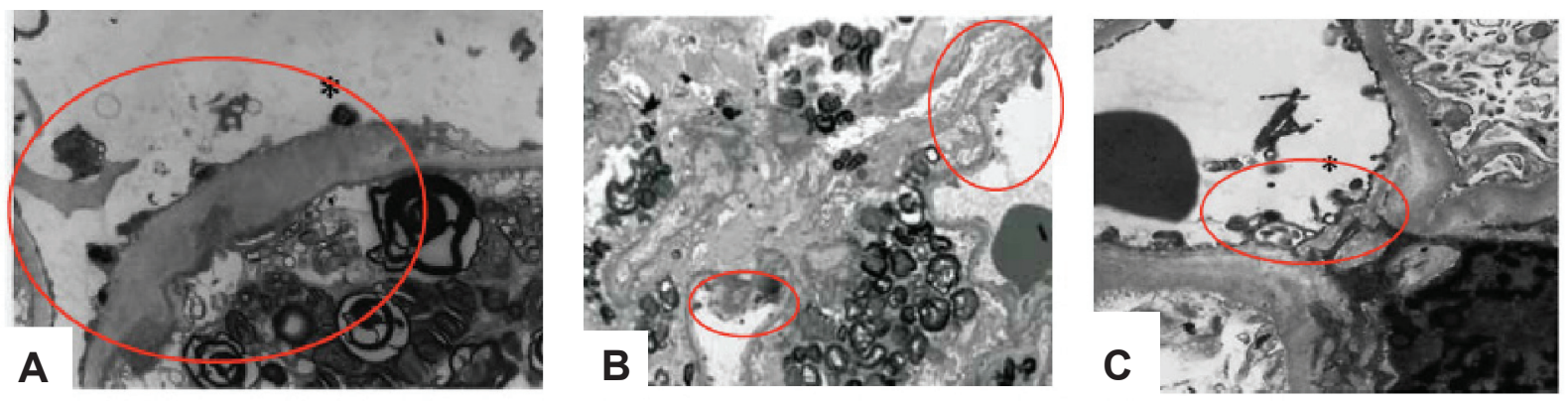

Figure 7 Glomerular capillary endothelial GL-3 deposits (circled) persist despite prolonged treatment with agalsidase-alfa at $0.2 \mathrm{mg} / \mathrm{kg}$ every 2 weeks. A) 38 year old male after 16 months of agalsidase-alfa at $0.2 \mathrm{mg} / \mathrm{kg}$ every other week, switched to agalsidase-beta after the biopsy; estimated glomerular filtration rate $47 \mathrm{~mL} / \mathrm{min} / 1.73 \mathrm{~m}$, urine protein $=0.55 \mathrm{~g} / 24 \mathrm{~h}$. This patient's clinic course is shown in Figure 7. B) 39 -year-old male after 24 months of agalsidase-alfa at $0.2 \mathrm{mg} / \mathrm{kg}$ every other week, switched to agalsidase-alfa at $0.4 \mathrm{mg} / \mathrm{kg}$ every 2 weeks after the biopsy; estimated glomerular filtration rate $34 \mathrm{~mL} / \mathrm{min} / 1.73 \mathrm{~m}^{2}$, urine protein $=2.7 \mathrm{~g} / 24 \mathrm{~h}$. This patient's clinic course is shown in Figure 8. C) 47 -year-old male after 60 months of agalsidase-alfa at $0.2 \mathrm{mg} / \mathrm{kg}$ every other week, switched to agalsidase-beta at $1.0 \mathrm{mg} / \mathrm{kg}$ every 2 weeks after the biopsy; estimated glomerular filtration rate $52 \mathrm{~mL} / \mathrm{min} / 1.73 \mathrm{~m}^{2}$, urine protein $=0.4 \mathrm{I} \mathrm{g} / 24 \mathrm{~h}$. This patient is the older brother of the patient shown in Figure 7 and Figure $9 \mathrm{~A}$. Panel A and C, adapted with permission from Warnock DG. 2005. Fabry disease: diagnosis and management, with emphasis on the renal manifestations. Curr Opin Nephrol Hypertens, 14:87-95. Copyright $\odot$ 2005. Panel B, adapted with permission from Torra R, Algaba F,Ars E, et al 2008. Preservation of renal function in a patient with Fabry nephropathy on enzyme replacement therapy. Clin Nephrol, 69:445-9. Copyright @ 2008 Dustri-Verlag. 

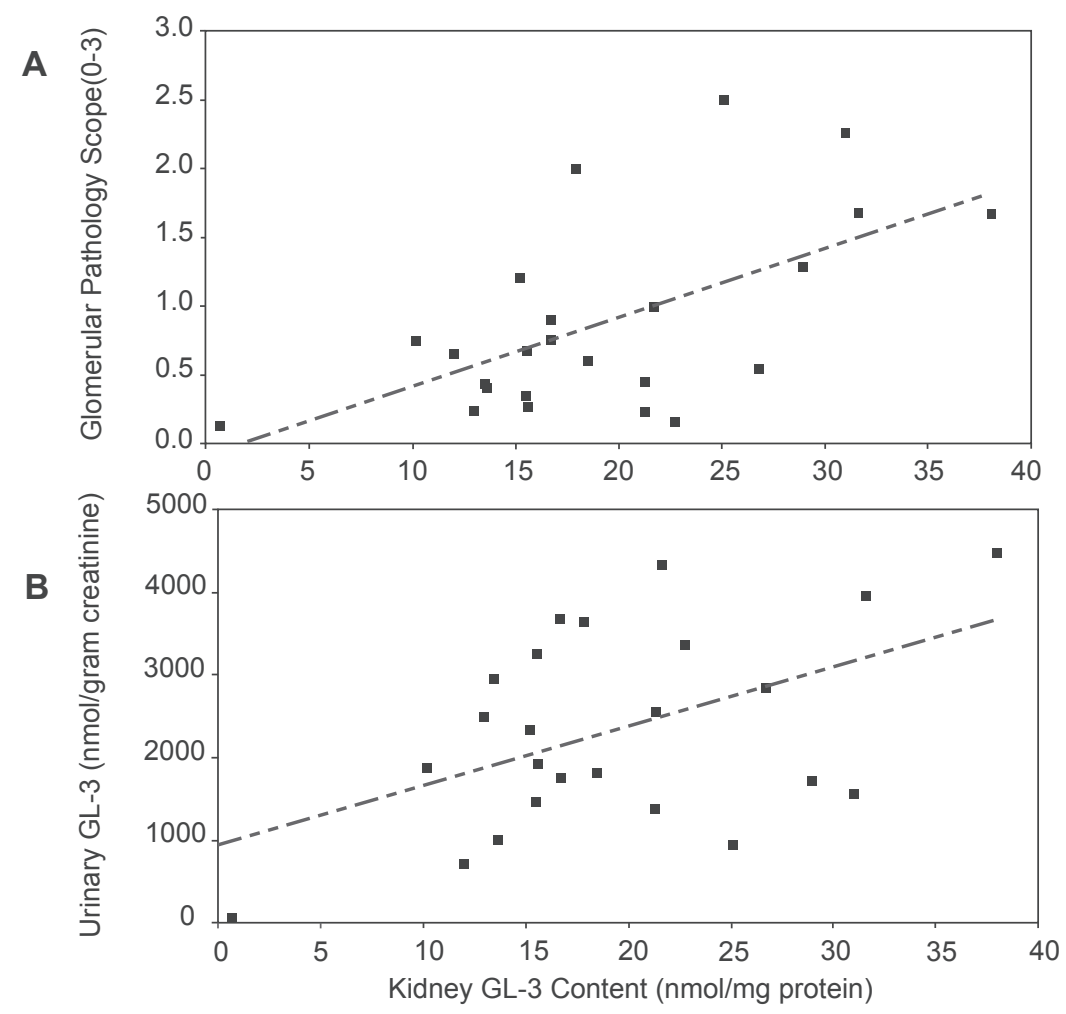

Figure 8 Relationship between kidney GL-3 content (nmol/mg protein) and Glomerular Pathology Scores A) and urinary GL-3 excretion (nmol/g creatinine) B) The linear regression shown in panel $A$ is $Y=0.505$ (SE 0.0145) $X-0.0959$ ( $S E 0.3027) ; F$ statistic $=0.0021 ; r^{2}=0.3554$. The linear regression shown in panel $B$ is $Y=71.0$ ( $S E$ 27.5) $X-964.8$ (SE 572.5); F statistic $=0.0169 ; r^{2}=0.2331$. Adapted with permission from Branton MH, Schiffmann R, Sabnis SG, et al 2002. Natural history of Fabry renal disease: influence of alpha-galactosidase A activity and genetic mutations on clinical course. Medicine (Baltimore), 8I:I22-38. Copyright @ 2002 Lippincott Williams \& Wilkins.

endothelial GL-3 clearance as a surrogate outcome measure for the clinical efficacy of ERT is, therefore not established at this time. Critical issues that have to be addressed include: A) Are the beneficial effects of ERT on clinical outcomes in Fabry nephropathy are reflected in concordant changes in GL-3 load? B) Are the underlying pathophysiologic processes improved in parallel with reductions in the GL-3 load? C) Are short-term changes in urinary GL-3 excretion of any utility is assessing response to ERT therapy? D) Do short-term changes in urinary GL-3 excretion reflect changes in the total body GL-3 load, or just changes in renal GL-3?

The phase IV study with agalsidase-beta (Banikazemi et al 2007) was designed with the knowledge that there was nearly complete microvascular endothelial GL-3 clearance with 1 year of ERT therapy. The primary outcome measures for the trial were chosen to be a composite of renal, cardiac and CNS events. The effect of ERT on the primary outcome measure did not reach statistical significance for the per-protocal analysis. Therefore, reduction in GL-3 inclusions did not predict clinical efficacy, and therefore resolution of GL-3 did not fulfill the criteria for a validated surrogate marker (Fleming 2005). The irony is that this surrogate marker may yet prove to be valid, but in this instance it was the clinical outcome study that fell short. The reasons for the lack of statistically significancant effects of ERT on the primary outcome measure have been reviewed (Schiffmann 2007), and include insufficient power of the study with only 82 participants, the differences in baseline proteinuria between the treatment and placebo group, and the failure to control protein excretion in either group with ACEI/ARB therapy. Despite these limitations of the phase IV study, Schiffmann concluded (Schiffmann 2007) that "...hemizygous male patients with the classic form of Fabry disease at possibly any age and symptomatic patients with milder variants should receive ERT with the particular goal to preserve renal function".

\section{Role of kidney biopsy in the management of Fabry nephropathy}

As previously mentioned, the role of kidney biopsy in new case discovery cannot be overemphasized. Even though Fabry disease may not have been considered in the differential diagnosis of a patient with CKD and proteinuria, The typical manifestations of Fabry nephropathy on biopsy 
are well described and readily appreciated, so the diagnosis should be readily discerned (Gubler et al 1978; Sessa et al 2002; Fervenza et al 2008).

In patients with a pre-established diagnosis of Fabry disease, there can still be an important role for kidney biopsy for assessing burden of disease, and assisting in problematic cases with decisions about when to initiate ERT, and conceivably dosing or frequency decisions (Ortiz et al 2008). It is too dogmatic to insist that every patient have a baseline biopsy, but several issues that need to be kept in mind. It is clear from the placebo-controlled trials with both forms of ERT, that patients with less severe "burden of disease" will do better on ERT than those with more advanced disease. There is no consensus, at present on the specific measures that should be included in the assessment of burden of disease, but likely factors would include decreased kidney function, proteinuria, pathologic changes on kidney biopsy, the extent and severity of GL-3 deposits. The magnitude of the baseline proteinuria (Banikazemi et al 2007; Germain et al 2007; Schiffmann et al 2007), the baseline level of GFR, and other conventional risk factors, such as hypertension, gender, smoking, and hypercholesterolemia have an impact on the burden of disease and rate of progression.

There are other progression factors that have not yet been defined. Kidney biopsy would seem to be worthwhile in such patients who have rapid progression without obvious risk factors for that progression. Based on the phase III results (Germain et al 2007), patients who have significant focal glomerulosclerosis and sclerosis are likely to progress quite rapidly. A similar finding was reported by Branton et al in which there appears to be a strong association between the baseline glomerulosclerosis scores, and the level of baseline proteinuria and GFR (Branton et al 2002). Even in young children, definite renal pathology has been described even in those who have not yet developed overt proteinuria or decreased GFR (Tondel et al 2008). In such cases, the kidney biopsy findings would favor institution of ERT rather than waiting for clinically evident target organ damage if the process is already established at the level of the biopsy.

It is essential that adequate tissue be obtained with sufficient number of glomeruli to assess overall involvement, including extent of focal and global glomerular fibrosis and sclerosis, and tubulo-interstitial fibrosis on light microscopy, and the extent of GL-3 deposits on the thin sections and electron microscopy (Valbuena et al 2008). While the typical "Zebra bodies" seen in podocytes on electron microscopy should lead to the correct diagnosis, the utility of scoring of the extent and severity of renal involvement are emerging as important factors in assessing prognosis and response to ERT and other therapies (Valbuena et al 2008). An international effort has been mounted to validate such a scoring system, with the hopes that it will be useful for describing the severity of disease and even response to therapy (Oliveira 2007).

Occasionally, there have been suggestions that ERT doses higher than $1 \mathrm{mg} / \mathrm{kg}$ might have some utility in treating refractory cases of Fabry nephropathy. There is simply not any evidence to support this proposal, especially considering the additional costs of what is already very expensive therapy. The results in Figures 4 and 5 suggest an approach that could be applied when ERT at doses greater than $1 \mathrm{mg} / \mathrm{kg}$ every 2 weeks is being considered. A kidney biopsy should be undertaken at this point (Ortiz et al 2008). There could be another form of kidney disease superimposed upon Fabry nephropathy that obviously would not respond to increased ERT, and in addition, if the biopsy demonstrates complete clearance of glomerular capillary endothelial GL-3 deposits, such as in Figure 7, then there does not appear to be any current justification for using a higher or more frequent dose of ERT. In contrast, the cases shown in Figure 6 had incomplete GL-3 endothelial clearance, and these patients were subsequently treated with higher doses of ERT than they received during their initial treatment period. This approach assumes that glomerular capillary endothelial GL-3 deposits are an acceptable marker for the burden of disease in Fabry nephropathy, an attractive hypothesis that has not yet been validated.

\section{Proteinuria and progression of Fabry nephropathy}

These reports do not establish an optimal dose for ERT in patients with moderately severe Fabry nephropathy, but are consistent with the hypothesis that proteinuria $>1 \mathrm{~g} /$ day is a major risk factor for progression regardless which dose or preparation is used. There may be additional factors, besides baseline eGFR and proteinuria, that drive progressive loss of kidney function, and need to be identified before the optimal dose and interval for ERT can be defined. Three reports have described patients with relatively low levels of baseline proteinuria ( $<0.5 \mathrm{~g} /$ day) before ERT was initiated; these reports are consistent with a possible effect of dose or frequency of dosing of ERT on the progression rate (Table 1). In the single case reported by Warnock (2005), the urine protein excretion was controlled with $\mathrm{ACEI} / \mathrm{ARB}$ therapy before agalsidase-alfa was started. During the 16-month agalsidase-alfa treatment period, the eGFR decreased at a rate of $-13.7 \mathrm{~mL} / \mathrm{min} / 1.73 \mathrm{~m}^{2} /$ year (Figure 5). Four patients from 
the Fabry Outcomes Survey Registry have been described with similar degrees of proteinuria during ERT (222.8 \pm $60[\mathrm{SEM}] \mathrm{mg} /$ day) who progressed at $-5.0 \pm 4.08 \mathrm{~mL} /$ $\min / 1.73 \mathrm{~m}^{2}$ per year on $0.2 \mathrm{mg} / \mathrm{kg}$ agalsidase-alfa given every 2 weeks (Patients 7, 10, 12 and 14; Table 5 [Schwarting et al 2006]). Schiffman et al (Schiffmann et al 2007) described 4 patients with low baseline proteinuria who progressed rapidly on $0.2 \mathrm{mg} / \mathrm{kg}$ agalsidase-alfa given every other week. The weighted average rate of progression for this group of 9 patients with low baseline proteinuria was $-6.69 \pm 2.90(\mathrm{SD}) \mathrm{mL} / \mathrm{min} / 1.73 \mathrm{~m}^{2} /$ year over a 27.6 month follow-up period. Table 1 also presents the data for 58 patients with low baseline proteinuria who received larger or more frequent doses of ERT than the 9 patients shown in the top section of Table 1 . The weighted average rate of progression for the group of 58 patients with low baseline proteinuria was $-0.97 \pm 2.10(\mathrm{SD}) \mathrm{mL} / \mathrm{min} / 1.73 \mathrm{~m}^{2} /$ year over a 45.2 month follow-up period. While these are simply open-label, uncontrolled observational studies, the results are consistent with the thesis that there is a subset of Fabry patients with low grade proteinuria who will continue to progress on agalsidase-alfa given at $0.2 \mathrm{mg} / \mathrm{kg}$ every other week, and who benefit by increasing the dose and/or frequency of ERT administration. It is work noting that the patient described by Warnock (Warnock 2005) and those described by Schiffman et al (2007) had slowing of their progressive loss of GFR when the ERT dose or frequency was increased above $0.2 \mathrm{mg} / \mathrm{kg}$ given every other week.

The importance of overt proteinuria as a major risk factor for progressive loss of kidney function was emphasized in the report of treatment outcomes from Würzburg (Breunig et al 2006), and proteinuria, as well as glomerular sclerosis and reduced baseline GFR were emphasized as risk factors for progression in the 54-month open label extension study of the Phase III study with agalsidase-beta (Germain et al 2007). Those patients who did not have overt proteinuria or significant glomerulosclerosis at baseline biopsy did quite well on ERT, while those with overt proteinuria ( $>1 \mathrm{~g} /$ day) and/or glomerular fibrosis or sclerosis in $50 \%$ of their glomeruli did not benefit from ERT at $1 \mathrm{mg} / \mathrm{kg}$ every 2 weeks (Germain et al 2007). Whether their outcome would have been improved if their proteinuria had been controlled with ACEI/ARB therapy is the subject of current investigation.

Proteinuria has emerged as a major determinant in the development of progressive tubular injury, interstitial fibrosis, and GFR loss in CKD (Remuzzi et al 2006), as well as Fabry nephropathy (Schiffmann 2007; Warnock 2007). Higher baseline levels of proteinuria are associated with more rapid decline of renal function (Breunig et al 2006; Banikazemi et al 2007; Germain et al 2007; Schiffmann et al 2007). The relationship between baseline proteinuria and the future occurrence of adverse kidney outcomes, even in patients with relatively mild Fabry nephropathy is depicted in Figure 9. At the initial assessment, the finding of significant proteinuria is a call for action, both for institution of effective ERT and control of proteinuria with ACEIs and ARBS. Elevated urinary protein excretion is a common finding in Fabry disease (Figure 1), and along with measurement of serum creatinine and calculation of eGFR, are integral elements of the clinical assessment of Fabry nephropathy (Ortiz et al 2008).

Four other patients have been described in the FOS Registry with marked degrees of proteinuria $(2.9 \pm 1.5[\mathrm{SEM}] \mathrm{g} /$ day $)$ but slow rates of progression $\left(0.38 \pm 1.52 \mathrm{~mL} / \mathrm{min} / 1.73 \mathrm{~m}^{2}\right.$ per year $)$ on $0.2 \mathrm{mg} / \mathrm{kg}$ agalsidase-alfa given every 2 weeks (Patients 1, 4, 17 and 20; Table 5 (Schwarting et al 2006)). This finding is at odds with all other reports of Fabry patients with similar baseline levels of GFR on any form of ERT who did not receive sufficient ACEI/ARB therapy to lower urine protein excretion to less than $1 \mathrm{~g}$ /day (Breunig et al 2006; Banikazemi et al 2007; Germain et al 2007; Schiffmann et al 2007).

The long-term results ( 54 months) for 58 patients who completed the phase III study of agalsidase-beta have recently been described. Following the initial 20-week double-blind phase, all 58 patients were transitioned to an open-label extension study, and received agalsidase-beta at $1 \mathrm{mg} / \mathrm{kg}$ every other week for as long as an additional 54 months (Germain et al 2007). Median serum creatinine and eGFR were relatively unchanged at the end of 54 months of open-label therapy for 42 patients whose baseline proteinuria was less than $1 \mathrm{~g} /$ day. The initial eGFR averaged $138 \mathrm{~mL} / \mathrm{min} / 1.73 \mathrm{~m}^{2}$, and the mean progression rate (eg, loss of eGFR) was $-1.005 \pm 0.970$ [SEM] $\mathrm{mL} / \mathrm{min} / 1.73 \mathrm{~m}^{2} /$ year (Figure 10 ). In contrast, 10 patients had rapid progression (rate $=-7.399 \pm 1,858$ [SEM] $\mathrm{m}: / \mathrm{min} / 1.73 \mathrm{~m}^{2} /$ year) despite relatively normal eGFR at baseline (average $=100 \mathrm{~mL} / \mathrm{min} / 1.73 \mathrm{~m}^{2}$ ). Two characteristics separated those with rapid progression from the majority who did not progress: the baseline proteinuria exceeded $1 \mathrm{~g} /$ day and the kidney biopsies revealed focal or global glomerular sclerosis in at least $50 \%$ of the glomeruli (Germain et al 2007).

It needs to be emphasized that ERT, by itself, has not been shown to reduce proteinuria in Fabry nephropathy. The phase III agalsidase-beta trial (Wilcox et al 2004) showed that the patients with overt proteinuria did not have any effect 


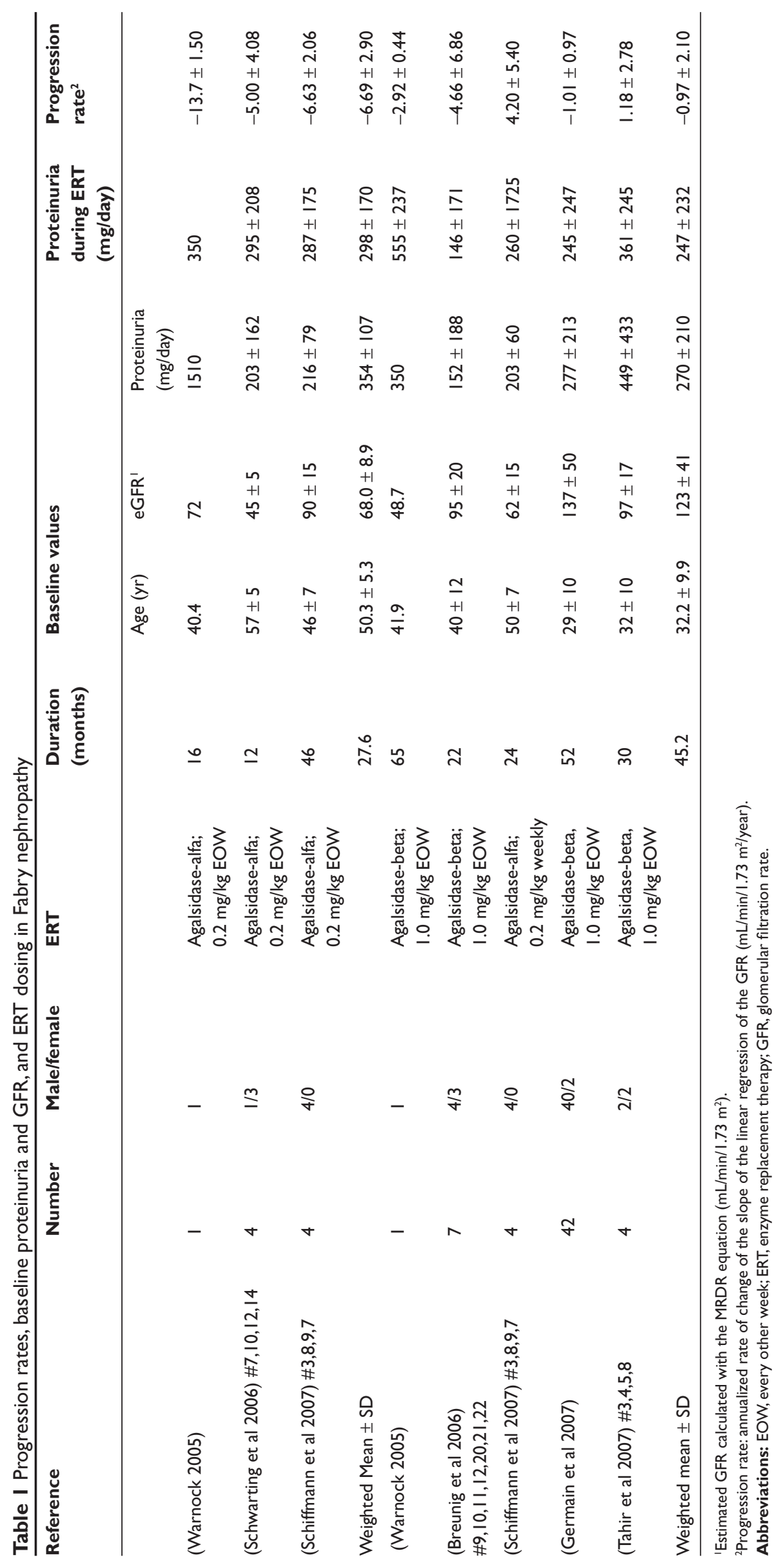




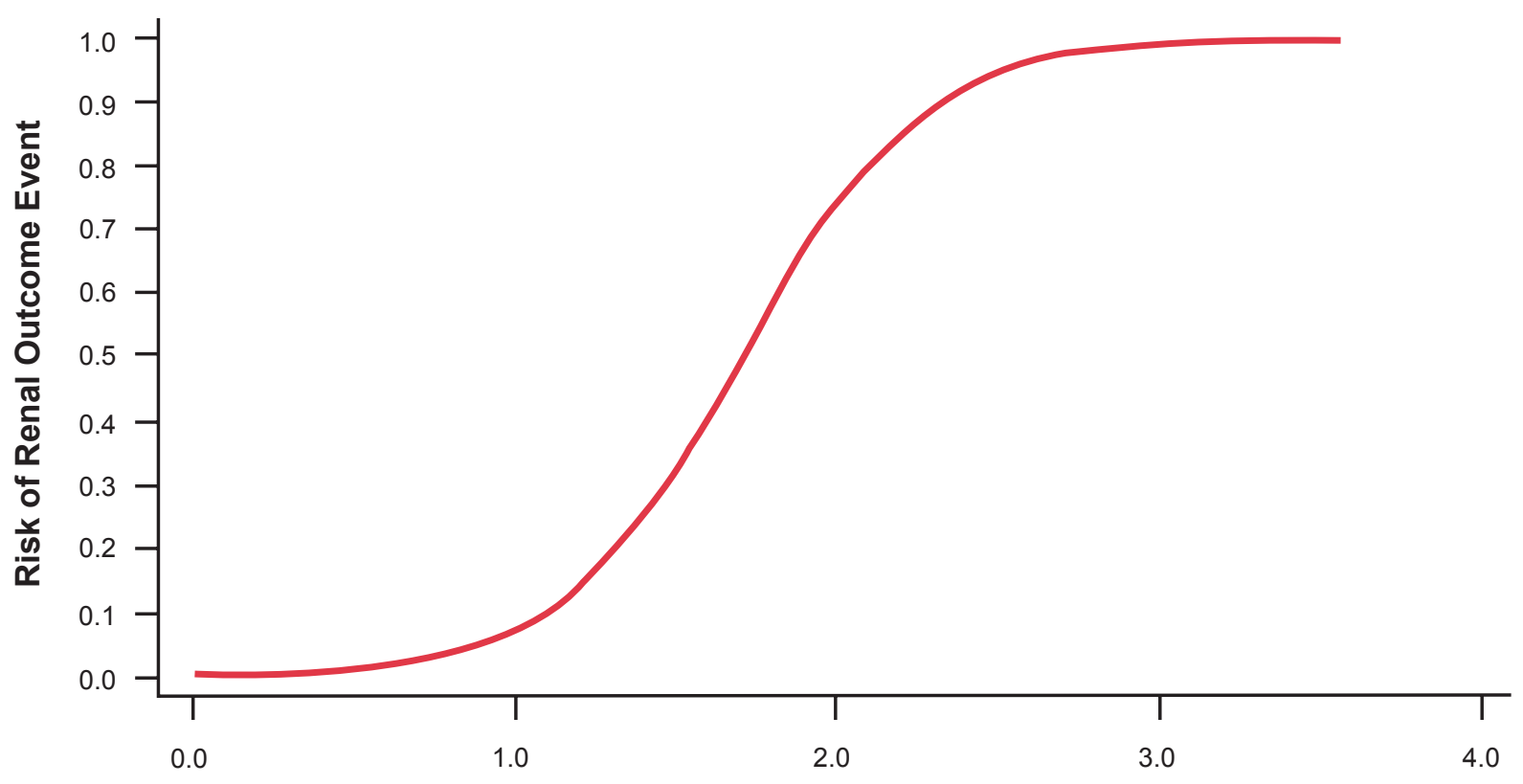

Years after Baseline Assessment

Figure 9 Relationship between baseline proteinuria and probability of a renal outcome event in the phase III extension study. Baseline proteinuria (expressed as the urinary protein/creatinine ratio) was determined before entry into the double-blinded initial phase of the study. Logistic regression analysis was used to determine the probability of a renal event (defined as $50 \%$ increase in serum creatinine compared to pre-treatment value, with the increased value $>1.4 \mathrm{mg} / \mathrm{dL}$ ). Adapted with permission from Germain $\mathrm{D}$, Waldek S, Banikazemi M, et al 2007. Sustained, long-term renal stabilization after 54 months of agalsidase beta therapy in patients with Fabry disease.J Am Soc Nephrol, I8: I547-57. Copyright (C) 2007 American Society of Nephrology.

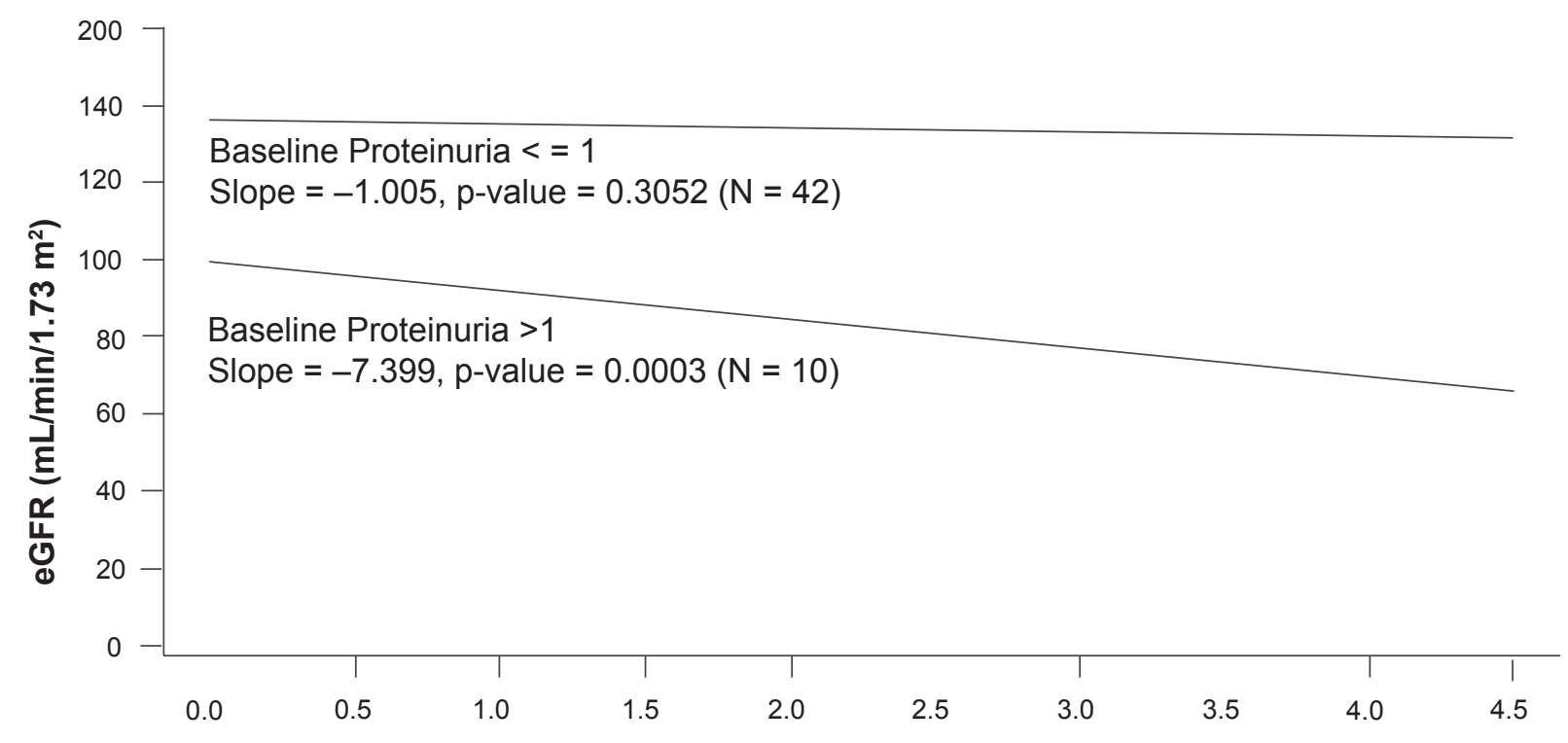

\section{Years After Starting ERT}

Figure 10 Relationship between baseline proteinuria and the rate of loss of estimated glomerular filtration rate (eGFR) in the phase III extension study. The participants were sub-grouped based on their baseline proteinuria, which was determined before entry into the double-blinded initial phase of the study. During the 54 -month treatment period, patients $(n=10)$ with baseline urinary protein/creatinine ratios $>1.0$ had a mean rate of decline of eGFR of $-7.4 \mathrm{~mL} / \mathrm{min}^{\prime} / .73 \mathrm{~m} / \mathrm{year}$. In contrast, patients $(\mathrm{n}=42)$ with baseline urinary protein/creatinine ratios $<1.0$ had a mean rate of decline of eGFR of $-1.0 \mathrm{~mL} / \mathrm{min} / 1.73 \mathrm{~m} / \mathrm{year}$. Adapted with permission from Germain D, Waldek S, Banikazemi M, et al 2007. Sustained, long-term renal stabilization after 54 months of agalsidase beta therapy in patients with Fabry disease. J Am Soc Nephrol, I8: I547-57. Copyright (C) 2007 American Society of Nephrology. 
of ERT on their level of proteinuria, and some patients with modest amounts of proteinuria at baseline developed overt proteinuria despite ERT. Similar results have been reported in the phase III extension study (Germain et al 2007), and in open label studies (Breunig et al 2006; Schiffmann et al 2006; Schiffmann et al 2007).

Previous studies had reported the use of ACEIs and/or ARBs in Fabry patients with proteinuria, but without any systematic approach to reduction of urinary protein excretion to a specific target (Wilcox et al 2004; Breunig et al 2006; Schiffmann et al 2006; Germain et al 2007; Schiffmann et al 2007). Because of the limited effect of ERT on slowing progression of Fabry nephropathy in patients with overt proteinuria at baseline, and the realization that the early success described in moderately severe Fabry nephropathy (Figure 2) was accompanied by the use on an ARB to control urine protein excretion, the systematic use of antiproteinuric therapy with ACEIs and/or ARBs was undertaken in a longterm open-label study of 10 patients (Tahir et al 2007). The study patients would historically have been at high risk for progressive loss of kidney function according to the phase III and phase IV results: baseline eGFR $<60 \mathrm{~mL} / \mathrm{min} / 1.73 \mathrm{~m}^{2}$, and baseline urine protein excretion $>1 \mathrm{~g}$ /day. When urine protein excretion was controlled to a target of $\leq 0.5 \mathrm{~g} /$ day by carefully titrating ACEI and ARB doses in these adult Fabry patients who were also treated with agalsidase-beta at $1 \mathrm{mg} / \mathrm{kg}$ every other week, the rate of loss of eGFR was not significantly different than zero (Tahir et al 2007). There was a decrease in blood pressure in these patients treated with anti-proteinuric therapy, which can limit dosing of ACEIs and ARBs. Fortunately, the doses required to control proteinuria in these patients were relatively modest (Tahir et al 2007). Although only observed in 6 patients, the slowing of progressive eGFR loss was much better than reported in the phase IV study for a similar group of patients with overt proteinuria and eGFR $<60 \mathrm{~mL} / \mathrm{min} / \mathrm{m}^{2}$ (Breunig et al 2006; Schiffmann et al 2006; Banikazemi et al 2007; Schiffmann et al 2007). In these previous series, the importance of baseline proteinuria was recognized, and a number of patients were treated with ACEI or ARB therapy, but there was not any systematic attempt to titrate the dose to achieve a targeted reduction in urine protein excretion to $\leq 0.5 \mathrm{~g} /$ day.

\section{Titration of urine proteinuria with anti-proteinuric therapy}

Data from the RENAAL study shows that the renal protective effect of losartan in Type II diabetic nephropathy was nearly fully explained by its anti-proteinuric effect
(Keane et al 2003; Eijkelkamp et al 2007). In both diabetic and nondiabetic patients with CKD, ACEIs and ARBs reduce proteinuria and slow progression, and are also cardio-protective (Mann et al 2008; ONTARGET 2008). It has long been appreciated that the degree of renal protection is related to the immediate reduction in proteinuria (GISEN 1997). The future rate of decline of GFR In patients with Fabry disease can be predicted from the baseline proteinuria at the time of initiation of ERT if urine protein excretion is not controlled (Figure 9). ERT alone will not reduce overt proteinuria in Fabry disease (Wilcox et al 2004; Banikazemi et al 2007; Germain et al 2007; Schiffmann 2007; Schiffmann et al 2007; Warnock 2007), but maintenance of urine protein excretion at $\leq 0.5 \mathrm{~g} /$ day with $\mathrm{ACEI} / \mathrm{ARB}$ therapy has been shown to stabilize eGFR in patients with moderately severe Fabry nephropathy who are receiving agalsidasebeta at $1 \mathrm{mg} / \mathrm{kg}$ every 2 weeks (De Schoenmakere et al 2003; Tahir et al 2007). Blood pressure is usually normal in Fabry patients (Wilcox et al 2004; Banikazemi et al 2007; Germain et al 2007; Schiffmann et al 2007; Tahir et al 2007). Thus, titration of anti-proteinuric agents is needed to the proteinuria target, rather than a systemic blood pressure target. Both telmisartan and ramipril were shown to slow progression in the ONTARGET Study, but worsened renal outcome when used in combination (Mann et al 2008). However, ONTARGET was a fixed-dose, forced titration study in patients with relatively modest proteinuria, while the approach proposed for Fabry nephropathy is titration of the proteinuria to a fixed target of $500 \mathrm{mg} /$ day. The hope is that ACEI and/or ARB therapy, together with optimal dosing of ERT will stabilize kidney function in patients with Fabry nephropathy, a clear-cut improvement over what has been reported in previous studies of Fabry patients who are at high risk of progressing to ESRD (Wilcox et al 2004; Breunig et al 2006; Banikazemi et al 2007; Germain et al 2007; Schiffmann et al 2007).

It appears that control of urine protein excretion is important in Fabry nephropathy, and that the optimal response to ERT requires the use of anti-proteinuric therapy to reduce the urine protein to a target of $\leq 0.5 \mathrm{~g} / \mathrm{day}$. This hypothesis is being tested in an ongoing, open-label, prospective multi-center observational trial that is called the FAACET Study (FAACET 2007); 40 patients with moderate to moderately severe Fabry nephropathy and overt proteinuria will be enrolled and followed for 24 months on combined therapy with agalsidase-beta at $1 \mathrm{mg} / \mathrm{kg}$ given every 2 weeks, and systematic titration of $\mathrm{ACEI} / \mathrm{ARB}$ therapy to reduce urine protein to $<500 \mathrm{mg} /$ day. It would be of great interest to see if a similar stabilization 
of eGFR would be observed in high risk Fabry patients with baseline eGFR $<60 \mathrm{~mL} / \mathrm{min} / 1.73 \mathrm{~m}^{2}$ and baseline proteinuria $>1 \mathrm{~g} /$ day who were treated with ACEI/ARB therapy to a target urine protein excretion of $<500 \mathrm{mg}$ /day while receiving agalsidase-alfa at $0.2 \mathrm{mg} / \mathrm{kg}$ every 2 weeks.

\section{Anti-agalsidase antibodies and the effectiveness of ERT}

The immunogenicity of infused exogenous agalsidase, and the subsequent generation of anti-agalsidase antibodies explains the infusion reactions that can occur after initiation of ERT in males who have no residual alpha-galactosidase activity. Such Infusion-associated events (rigors, fever, myalgias) are related to infused dose and infusion rate, but rarely can be attributed to true IgE-related anaphylaxis (Schiffmann et al 2001; Wilcox et al 2004; Banikazemi et al 2007). Even with severe reactions, it now appears possible to de-sensitize patients who develop $\operatorname{IgE}$ responses to agalsidase-beta (Bodensteiner et al 2008).

Antibody responses against agalsidase are rarely encountered in females, who generally have some circulating enzyme and residual alpha-galactosidase A activity (Fuller et al 2004). If they do develop anti-agalsidase antibodies, the titers are generally low, and in the long run, they appear to become tolerant to ERT (Banikazemi et al 2007). There may also be an effect of the underlying mutation; entire deletions and early stop-codons result in the absence of circulating gene product. On the other hand, mis-sense deletions or frame-shifts can result in circulating proteins that may have native native alpha-galactosidase A sequence in the N-terminus, while the rest of the protein is unrelated to the native protein. Most male patients with Fabry disease are negative for cross-reacting immunologic material (CRIM) (Wilcox et al 2004). Patients who are CRIM-positive do not appear to have immunologic reactions if their mutations do not impair antigen presentation during the development of self-tolerance during the late fetal and post-natal period (Kyewski and Klein 2006).

Despite differences in glycosylation that could affect tissue delivery and uptake (Lee et al 2003), agalsidase-alfa and agalsidase-beta appear have identical immunogenicity and specific activity (Lee et al 2003; Linthorst et al 2004). There is a single report from Japan describing markedly reduced specific enzyme activity of agalsidase-alfa compared with agalsidase-beta (Sakuraba et al 2006), but this finding has not been confirmed by other reports.

In vitro studies have shown that anti-agalsidase antibodies can exhibit neutralizing capacity towards the infused enzyme. In addition, there is accumulating evidence in a number of lysosomal storage disorders that antibodies generated against infused recombinant enzyme can also interfere with mannose-6-phosphate-mediated receptor uptake, even though the antibodies may not affect the specific activity of the enzyme. Thus, there are two distinct mechanisms that can impair the effectiveness of infused enzyme (Wang et al 2008). In vivo, patients with anti-agalsidase IgG have blunted urinary GL-3 clearance, as compared to those who remain antibody-negative (Linthorst et al 2004; Whitfield et al 2005). The prevalence and titers of anti-agalsidase antibodies do not differ between patients treated with different doses $(0.2 \mathrm{mg} / \mathrm{kg}$ versus $1.0 \mathrm{mg} / \mathrm{kg}$ body weight every 2 weeks) of ERT or in patients switched from lower to higher doses of ERT (Vedder et al 2008).

The issue of antibody titers was addressed in a recent study that compared the two different preparations of ERT (either agalsidase-alfa or agalsidase-beta at $0.2 \mathrm{mg} / \mathrm{kg}$ every 2 weeks) versus agalsidase-beta at $1.0 \mathrm{mg}$ (Vedder et al 2008). There were no significant differences in the prevalence of neutralizing antibodies in patients treated with $0.2 \mathrm{mg} / \mathrm{kg}$ of agalsidase-alfa compared to agalsidase-beta, or in patients treated with $0.2 \mathrm{mg} / \mathrm{kg}$ of agalsidase-beta compared with $1 \mathrm{mg} / \mathrm{kg}$ agalsidase-beta (Vedder et al 2008). More complete clearance of plasma and urinary GL-3 in male patients treated with the $1.0 \mathrm{mg} / \mathrm{kg}$ agalsidase-beta was observed than in those treated with either preparation at $0.2 \mathrm{mg} / \mathrm{kg}$ every 2 weeks. Of note, the reduction in left ventricular mass was similar in antibody-positive and antibody-negative patients, as long as they were treated with ERT at $1 \mathrm{mg} / \mathrm{kg}$ every 2 weeks (Vedder et al 2008).

These results have been criticized (Mehta et al 2008) for the small number of patients, and for pooling the results for patients treated with $0.2 \mathrm{mg} / \mathrm{kg}$ agalsidase-alfa with the results for patients treated with $0.2 \mathrm{mg} / \mathrm{kg}$ agalsidase-beta. It was asserted that the two ERT preparations not identical, despite the previous reports that the two preparations have identical immunogenecity (Lee et al 2003; Linthorst et al 2004). The assessment of cardiac improvement was limited to measurements of left ventricular mass, without clear-cut reference to baseline values or improvement in other measures of cardiac function. Urinary clearance of GL-3 was impaired in antibody-positive patients treated with $0.2 \mathrm{mg} / \mathrm{kg}$ ERT, confirming previous observations (Linthorst et al 2004; Schiffmann et al 2006), but this finding has not been shown to correlate with any differences in clinical outcomes in prospective studies. In fact, kidney function remained stable for 12 months in all of the treatment groups in the study (Vedder et al 2008). 
In vitro studies in mice with sera from anti-agalsidase antibody-positive patients (Ohashi et al 2008; Vedder et al 2008). and in vivo studies in patients (Vedder et al 2008) suggest that the potential neutralizing effects of anti-agalsidase antibodies may be overcome by increasing the dose or ERT from 0.2 to $1.0 \mathrm{mg} / \mathrm{kg}$. It is not known whether the effects or titers of anti-agalsidase neutralizing IgG antibodies correlate with long-term clinical outcomes, but this point clearly needs to be addressed. The increase in urinary GL-3 excretion above baseline in antibody-positive patients treated with ERT at $0.2 \mathrm{mg} / \mathrm{kg}$ every other week only became apparent after 6 months of infusion therapy, was not correlated with infusion-related reactions. No information is available about doses other than 0.2 or $1.0 \mathrm{mg} / \mathrm{kg}$ ERT, or for dosing intervals other than every other week. For now, it would seem prudent to regularly monitor urinary GL-3 excretion, especially in patients treated with ERT at $0.2 \mathrm{mg} / \mathrm{kg}$ every 2 weeks. If there is a progressive rise in urinary GL-3 excretion after several months of ERT, then consideration should be given to increasing the frequency of monitoring of clinical outcomes. If there is any subsequent indication of decreasing eGFR, or increasing proteinuria, then increasing the dose of ERT to $1 \mathrm{mg} / \mathrm{kg}$ every other week could be considered. If there are continuing and severe infusion-related reactions, then de-sensitization (Bodensteiner et al 2008), or even induction of tolerance (Wang et al 2008) may be worthwhile. Increasing urinary GL-3 excretion has not been associated with any adverse clinical outcome at this time; more work is required to understand the clinical significance of this finding, and to determine if urinary GL-3 excretion can serve as a useful biomarker that is related to the whole body disease burden and responses to therapies.

\section{ERT in special populations Pediatric patients with Fabry disease}

The mean age of symptom onset of Fabry disease has been reported to be 10 years in boys and 15 years in girls, with cases presenting as early as 3 years in boys and 6 years in girls, and renal failure developing as early as 16 years of age in boys (MacDermot et al 2001; Ries et al 2003; Desnick and Brady 2004; Ries et al 2006). In the Fabry Outcome Survey database, 8 patients had albuminuria and 12 patients had proteinuria from a total of 82 patients younger than 18 years (Ramaswami et al 2006). A recent report from the Fabry Registry of 352 pediatric patients (age $<18$ years) described 2 children with Stage 2 CKD, and 1 child with stage $3 \mathrm{CKD}$, indicating that some Fabry patients develop significant nephropathy during childhood (Hopkin et al 2008).
Twenty-four hour urine assessments were available in 86 patients; 4 males and 5 females had urinary protein excretion $>150 \mathrm{mg} / 24 \mathrm{~h}$. Four additional patients ( 3 males and 1 female) had albumin excretion rates $>30 \mathrm{mg} / 24 \mathrm{~h}$ among the 46 patients for whom these data were available.

In contrast to the well-described kidney biopsy findings in adult patients, there is limited information concerning renal morphological changes in young patients with Fabry disease. Renal GL-3 deposits have been described as early as the first 5 months of pregnancy (Brady et al 1971; Elleder et al 1998). GL-3 deposits were described in renal biopsies from 3 children aged 8 (female), 11, and 12 years (males), all without proteinuria (Gubler et al 1978); GL-3 deposits were detected at an early age in vessels and glomeruli in male patients, whereas the distribution of GL-3 deposits was more irregular in female patients (Gubler et al 1978). A series was reported of kidney biopsies in 7 male and 2 female pediatric patients with a mean age of 13.5 years (range, 7 to 18 years); biopsies were performed before the start of ERT in 7 patients, and after 2 years of ERT in 2 patients (Tondel et al 2008). In all patients, light and electron microscopy showed severe GL-3 accumulation in podocytes. A conspicuous and worrisome finding was the presence of hyaline deposits in the media of small renal arteries in nearly half (4 of 9) these pediatric patients who underwent biopsy before they developed overt clinical findings of Fabry nephropathy (Tondel et al 2008).

In these biopsy studies, significant deposition of GL-3 was found in glomeruli, as well as the tubulo-interstitial and vascular compartments long before overt Fabry nephropathy was manifested clinically (Gubler et al 1978; Tondel et al 2008; Valbuena et al 2008). These observations demonstrate that albuminuria or overt proteinuria are not reliable early predictors of Fabry nephropathy since glomerulosclerosis as well as significant tubulo-interstitial fibrosis can occur before overt proteinuria or significant loss of kidney function occurs. While there is ongoing debate about the optimal timing for initiating ERT, especially in pediatric patients, the recent biopsy studies argue against waiting until there is demonstrated kidney damage as evidenced by reduced eGFR or overt proteinuria before initiating ERT.

There are important concerns about the accurate measurement of GFR in Fabry patients. In the recent study from the Fabry Registry (Hopkin et al 2008), the majority of children had estimated GFR values (using the Schwartz formula) that were higher than expected for healthy children and adolescents of the same age. The study by Tøndel et al supports these observations in that there was a significant increase in eGFR when compared with iohexol-GFR in all 
patients in the study group (Tøndel et al 2008), a finding that has been referred to as "hyperfiltration" (Rodriguez-Iturbe et al 1985). Clearly, accurate and validated measurements of GFR are needed, especially in children if this information is going to be used as a criterion for initiating ERT or following the response to ERT in the individual patient.

There are very few studies of the effects of ERT in pediatric populations. Ries et al conducted a prospective study of ERT for 6 months in 24 children (mean age, 11.8 years; range, 6.5-18 years) and showed that treatment was safe and well tolerated (Ries et al 2006). In this study, renal hyperfiltration was corrected, albuminuria decreased in 3 of 4 subjects, and heart rate variability improved, indicating that there may be a "window of opportunity" to correct early organ damage and prevent progressive disease with early initiation of ERT (Ries et al 2006). Similarly, in the study by Tøndel et al electron microscopy showed glomerular endothelial GL-3 inclusions, in all patients except the 2 boys treated with agalsidase-alfa at $0.2 \mathrm{mg} / \mathrm{kg}$ every 2 weeks for 2 years (Tondel et al 2008). On the other hand, these two boys developed de novo albuminuria, glomerulosclerosis, interstitial fibrosis and podocyte GL-3 deposits, while they were being treated with ERT at a dose of $0.2 \mathrm{mg} / \mathrm{kg}$ (Tondel et al 2008).

Wraith et al described 14 male and 2 female patients, 8 to 16 years old, who received an open-label 48 -week course of agalsidase-beta at $1 \mathrm{mg} / \mathrm{kg}$ infused intravenously every 2 weeks (Wraith et al 2008). ERT was shown to reduce GL-3 accumulation in dermal endothelium in children with Fabry disease (Wraith et al 2008). Mild proteinuria, defined as $>100 \mathrm{mg} / \mathrm{m}^{2} / 24 \mathrm{~h}$ for pediatric patients (Hogg et al 2000), was seen in 8 of 15 patients at baseline, and 3 of the 8 patients had values $\geq 100 \mathrm{mg} / \mathrm{m}^{2} / 24 \mathrm{~h}$ at week 48 . Mean eGFR was $126 \pm 29(\mathrm{SD}) \mathrm{mL} / \mathrm{min} / 1.73 \mathrm{~m}^{2}$ at baseline $(\mathrm{n}=16)$, and $125 \pm 26(\mathrm{SD})$ at week $48(\mathrm{n}=15)$. For the 3 adolescent male patients with possible hyperfiltration and mild proteinuria at baseline, further deterioration of renal function was not observed during the 48 months of follow-up. The overall safety profile for agalsidase-beta in pediatric patients is similar to that observed in adult patients (Eng et al 2001; Banikazemi et al 2007; Germain et al 2007; Wraith et al 2008).

The pediatric studies published to date have not included enough patients with sufficient long-term follow up to determine whether early initiation of ERT can prevent the development of irreversible target-organ damage, or just slow the progression of disease. Prospective treatment trials and long-term monitoring of a significant number of patients are required to determine the impact of ERT on this group of patients. An ongoing open-label study (FIELD 2008) is currently examining the use of agalsidase-beta given at $1 \mathrm{mg} / \mathrm{kg}$ every 4 weeks compared to $0.5 \mathrm{mg} / \mathrm{kg}$ given every 2 weeks in a group of 24 males less than 18 years of age with minimal symptoms; the primary outcome measure is clearance of GL-3 deposits in dermal vascular endothelial cells. The secondary outcome measures include reductions in plasma and urinary GL-3 levels.

\section{Kidney transplant recipients with Fabry disease}

ESRD in Fabry disease can be successfully managed by kidney transplantation (Ojo et al 2000). The pharmacokinetics of agalsidase-alfa is the same in transplant patients as in Fabry patients with native kidneys (Pastores et al 2007). The detection of Fabry patients among dialysis and transplant patients is important because of organs other than the kidneys are involved in Fabry disease. The hope is that ERT treatment may ameliorate symptoms or prevent further progression of cardiovascular and cerebrovascular complications in these patients. A pilot study reported improvement in left ventricular hypertrophy and ejection fraction in 3 Fabry patients who had been transplanted and were treated with agalsidase-beta at $1 \mathrm{mg} / \mathrm{kg}$ every other week (Mignani et al 2004). These preliminary findings have been extended and confirmed in a larger group of patients transplanted with an average follow-up of 48 months on ERT after kidney transplantation (Mignani et al 2008).

\section{ERT in dialysis patients}

Administration of ERT during hemodialysis is not associated with a reduction of the enzyme activity or enzyme loss into the dialysate of agalsidase-alfa (Pastores et al 2007), or agalsidase-beta (Kosch et al 2004). Agalsidase-beta given to Fabry ESRD patients on hemodialysis was associated with improvement in pain and gastrointestinal symptoms, and halved the rate of progression of left ventricular mass index compared with the rate before institution of ERT (Pisani et al 2005), although there is a suggestion that the cardiac response to ERT may not be quite as favorable in Fabry patients on dialysis as has been described in Fabry patients who have received kidney transplants (Mignani et al 2008).

\section{Conclusions and pending issues}

While the "classical" presentation of Fabry disease is well described (Desnick et al 2001; Branton et al 2002), there are patients who have serious organ involvement without all of the classical findings. The phenotypic variation in females, and even among family members with the same 
AGAL mutation is notable; additional work is needed to better define the basis for this variation. The appreciation of the wider spectrum of disease than is described by the classical phenotype is important since the clinical diagnosis may not be apparent in patients who lack the typical signs and symptoms of Fabry disease, and for whom the appropriate diagnostic testing is not carried out.

The advent of ERT has transformed Fabry disease into a treatable cause of proteinuric CKD. However, some issues need to be addressed:

- The general applicability of anti-proteinuria therapy in the context of ERT therapy for Fabry nephropathy needs to be confirmed and extended to a large cohort of patients. Both the optimal target for urine protein reduction, and the optimal dose of ERT in patients who have had their proteinuria reduced to that target, need to be defined.

- In patients with baseline proteinuria $>1 \mathrm{~g} /$ day, the prospects need to be determined for stabilization of kidney function with approved doses of agalsidasealfa or agalsidase-beta plus titration of proteinuria to less than $500 \mathrm{mg}$ /day with ACEI/ARB therapy.

○ In patients with baseline proteinuria $<1 \mathrm{~g}$ /day, the prospects need to be determined for stabilization of kidney function with approved doses of agalsidase-alfa or agalsidase-beta plus minimization of proteinuria with ACEI/ARB.

- The therapeutic benefits of ERT on cerebrovascular and cardiovascular events remain to be demonstrated in outcome studies that are not dominated by the occurrence of renal events. Transplant and dialysis patients, and patients in whom kidney function has been stabilized with ERT and control of proteinuria with ACEI/ARB therapy would be appropriate subjects for these prospective studies.

- Just as anti-proteinuric therapy appears to be important adjuncts for ERT in Fabry nephropathy, the usefulness of similar adjuncts to ERT for the cardiovascular and cerebrovascular manifestations of Fabry disease need to be explored. It is notable that some Fabry patients who have had stabilization of their kidney function on ERT can still develop cardiovascular complications of the disease, so ACEI/ARB therapy may not be as cardio-protective in Fabry patients as in other patient cohorts.

- The optimal timing of initiation of ERT before irreversible organ damage has occurred needs to be addressed.

The available evidence strongly supports the provision of ERT for Fabry nephropathy, even though the current expectation is to halt progression of disease, rather than any actual resolution of pre-existent organ damage. Rather than being a criticism to the effectiveness of ERT in Fabry nephropathy, it should be recognized that this limitation exists for all forms of CKD, emphasizing the importance of surveillance, early detection and close monitoring of burden of disease and progression of organ involvement as important contributors to the ultimate success of treating these diseases.

\section{Contribution made by each author}

The authors have prepared this manuscript in its entirety; the services of a medical writer were not used at any point in the preparation of this work. The manuscript did not undergo any sort of "pre-review" by any pharmaceutical company or outside agency. The authors are fully responsible for contents and editorial decisions for this manuscript.

\section{Acknowledgment}

We thank Dr Gabor Linthorst (Amsterdam Medical Center) for his helpful suggestions and review of the sections dealing with anti-agalsidase antibodies.

\section{Disclosures}

Dr Fervenza has received travel support and honoraria for lectures from Genzyme Corporation and Shire Corporation.

Dr Torra has received travel support and honoraria for lectures from Genzyme Corporation and Shire Corporation.

Dr Warnock is a member of the North American Advisory Board of the Fabry Registry, a consultant for Genzyme Corporation on Fabry disease, and has received honoraria for lectures and research grants from Genzyme Corporation.

\section{References}

Aerts JM, Groener JE, Kuiper S, et al. 2008. Elevated globotriaosylsphingosine is a hallmark of Fabry disease. Proc Natl Acad Sci U S A, 105:2812-7.

Askari H, Kaneski CR, Semino-Mora C, et al. 2007. Cellular and tissue localization of globotriaosylceramide in Fabry disease. Virchows Arch, 451:823-34.

Banikazemi M, Bultas J, Waldek S, et al. 2007. Agalsidase-beta therapy for advanced Fabry disease: a randomized trial. Ann Intern Med, 146:77-86.

Barbey F, Lidove O, Schwarting A. 2008. Fabry nephropathy: 5 years of enzyme replacement therapy - a short review. Nephrol Dial Transplant Plus, 1:11-9.

Blom D, Speijer D, Linthorst GE, et al. 2003. Recombinant enzyme therapy for Fabry disease: absence of editing of human alpha-galactosidase A mRNA. Am J Hum Genet, 72:23-31.

Bodensteiner D, Scott CR, Sims KB, et al. 2008. Successful reinstitution of agalsidase beta therapy in Fabry disease patients with previous IgE-antibody or skin-test reactivity to the recombinant enzyme. Genet Med, 10:353-8.

Brady RO, Gal AE, Bradley RM, et al. 1967. Enzymatic defect in Fabry's disease. Ceramidetrihexosidase deficiency. $N$ Engl J Med, 276:1163-7. 
Brady RO, Uhlendorf BW, Jacobson CB. 1971. Fabry’s disease: antenatal detection. Science, 172:174-5.

Branton MH, Schiffmann R, Sabnis SG, et al. 2002. Natural history of Fabry renal disease: influence of alpha-galactosidase A activity and genetic mutations on clinical course. Medicine (Baltimore), $81: 122-38$.

Breunig F, Weidemann F, Strotmann J, et al. 2006. Clinical benefit of enzyme replacement therapy in Fabry disease. Kidney Int, 69:1216-21.

De Schoenmakere G, Chauveau D, Grunfeld JP. 2003. Enzyme replacement therapy in Anderson-Fabry's disease: beneficial clinical effect on vital organ function. Nephrol Dial Transplant, 18:33-5.

Deegan PB, Baehner AF, Barba Romero MA, et al. 2006. Natural history of Fabry disease in females in the Fabry Outcome Survey. $J$ Med Genet, 43:347-52.

Desnick R, Ioannou Y, Eng C. 2001. In: Scriver C, Beaudet A, Sly W, et al. (eds). Alpha-galactosidase A deficiency: Fabry disease. the metabolic bases of inherited disease. New York, McGraw-Hill. p. 3733-74.

Desnick RJ. 2004. Enzyme replacement therapy for Fabry disease: lessons from two alpha-galactosidase A orphan products and one FDA approval. Expert Opin Biol Ther, 4:1167-76.

Desnick RJ, Brady RO. 2004. Fabry disease in childhood. J Pediatr, 144:S20-6.

Eijkelkamp WBA, Zhang Z, Remuzzi G, et al. 2007. Albuminuria is a target for renoprotective therapy independent from blood pressure in patients with type 2 diabetic nephropathy: post hoc analysis from the reduction of endpoints in NIDDM with the Angiotensin II Antagonist Losartan (RENAAL) Trial. J Am Soc Nephrol, 18:1540-6.

Elleder M, Poupetova H, Kozich V. 1998. [Fetal pathology in Fabry's disease and mucopolysaccharidosis type I]. Cesk Patol, 34:7-12.

Eng CM, Banikazemi M, Gordon RE, et al. 2001. A phase 1/2 clinical trial of enzyme replacement in Fabry disease: pharmacokinetic, substrate clearance, and safety studies. Am J Hum Genet, 68:711-22.

Eng CM, Guffon N, Wilcox WR, et al. 2001. Safety and efficacy of recombinant human alpha-galactosidase A - replacement therapy in Fabry's disease. $N$ Engl J Med, 345:9-16.

FAACET. 2007. The Fabrazyme ${ }^{\circledR}$ and Arbs and ACE inhibitor treatment (FAACET) Study (NCT00446862). Accessed September 9, 2007. URL: http://www.clinicaltrials.gov/ct/search; jsessionid $=87$ 169CD1104ADB522E4B53168DEA01DD?term=NCT00446862\& submit=Search

Fabry H. 2001. An historical overview of Fabry disease. J Inherit Metab Dis, 24(Suppl 2):3-7.

Fervenza FC, Torra R and Lager DJ. 2008. Fabry disease: an underrecognized cause of proteinuria. Kidney Int, 73:1193-9.

FIELD. 2008. A study of two Fabrazyme ${ }^{\circledR}$ dosing regimens in treatmentnaïve male pediatric patients without severe symptoms (NCT00701415). Accessed August 14, 2008. URL: http://www.clinicaltrials.gov/ct2/ show/NCT00701415?term=FAbry\&rank=42.

Fleming TR. 2005. Surrogate endpoints and FDA's accelerated approval process. Health Aff (Millwood), 24:67-78.

Fuller M, Lovejoy M, Brooks DA, et al. 2004. Immunoquantification of alpha-galactosidase: evaluation for the diagnosis of Fabry disease. Clin Chem, 50:1979-85.

Germain D, Waldek S, Banikazemi M, et al. 2007. Sustained, long-term renal stabilization after 54 months of agalsidase beta therapy in patients with Fabry disease. J Am Soc Nephrol, 18:1547-57.

Germain DP. 2001. A new phenotype of Fabry disease with intermediate severity between the classical form and the cardiac variant. Contrib Nephrol, 136:234-40.

GISEN. 1997. Randomised placebo-controlled trial of effect of ramipril on decline in glomerular filtration rate and risk of terminal renal failure in proteinuric, non-diabetic nephropathy. The GISEN Group (Gruppo Italiano di Studi Epidemiologici in Nefrologia). Lancet, 349:1857-63.

Gubler MC, Lenoir G, Grunfeld JP, et al. 1978. Early renal changes in hemizygous and heterozygous patients with Fabry's disease. Kidney Int, 13:223-35.
Gupta S, Ries M, Kotsopoulos S, et al. 2005. The relationship of vascular glycolipid storage to clinical manifestations of Fabry disease: a crosssectional study of a large cohort of clinically affected heterozygous women. Medicine (Baltimore), 84:261-8.

Hauser AC, Lorenz M, Sunder-Plassmann G. 2004. The expanding clinical spectrum of Anderson-Fabry disease: a challenge to diagnosis in the novel era of enzyme replacement therapy. J Intern Med, 255:629-36.

Hogg RJ, Portman RJ, Milliner D, et al. 2000. Evaluation and management of proteinuria and nephrotic syndrome in children: recommendations from a pediatric nephrology panel established at the National Kidney Foundation conference on proteinuria, albuminuria, risk, assessment, detection, and elimination (PARADE). Pediatrics, 105:1242-9.

Hopkin RJ, Bissler J, Banikazemi M, et al. 2008. Characterization of Fabry disease in 352 pediatric patients in the Fabry Registry. Pediatr Res, 64:550-5.

Kaneski CR, Moore DF, Ries M, et al. 2006. Myeloperoxidase predicts risk of vasculopathic events in hemizgygous males with Fabry disease. Neurology, 67:2045-7.

Keane WF, Brenner BM, de Zeeuw D, et al. 2003. The risk of developing end-stage renal disease in patients with type 2 diabetes and nephropathy: the RENAAL study. Kidney Int, 63:1499-507.

Kobayashi M, Ohashi T, Sakuma M, et al. 2008. Clinical manifestations and natural history of Japanese heterozygous females with Fabry disease. $J$ Inherit Metab Dis.

Kosch M, Koch HG, Oliveira JP, et al. 2004. Enzyme replacement therapy administered during hemodialysis in patients with Fabry disease. Kidney Int, 66:1279-82.

Kyewski B, Klein L. 2006. A central role for central tolerance. Annu Rev Immunol, 24:571-606.

Lee K, Jin X, Zhang K, et al. 2003. A biochemical and pharmacological comparison of enzyme replacement therapies for the glycolipid storage disorder Fabry disease. Glycobiology, 13:305-13.

Linthorst GE, Hollak CE, Donker-Koopman WE, et al. 2004. Enzyme therapy for Fabry disease: neutralizing antibodies toward agalsidase alpha and beta. Kidney Int, 66:1589-95.

MacDermot KD, Holmes A, Miners AH. 2001. Anderson-Fabry disease: clinical manifestations and impact of disease in a cohort of 98 hemizygous males. J Med Genet, 38:750-60.

Mann JF, Schmieder RE, McQueen M, et al. 2008. Renal outcomes with telmisartan, ramipril, or both, in people at high vascular risk (the ONTARGET study): a multicentre, randomised, double-blind, controlled trial. Lancet, 372:547-53.

Meehan SM, Junsanto T, Rydel JJ, et al. 2004. Fabry disease: renal involvement limited to podocyte pathology and proteinuria in a septuagenarian cardiac variant. Pathologic and therapeutic implications. Am J Kidney Dis, 43:164-71.

Mehta A, Beck M, Kampmann C, et al. 2008. Enzyme replacement therapy in Fabry disease: Comparison of agalsidase alfa and agalsidase beta. Mol Genet Metab, 95:114-5.

Mehta A, Ricci R, Widmer U, et al. 2004. Fabry disease defined: baseline clinical manifestations of 366 patients in the Fabry Outcome Survey. Eur J Clin Invest, 34:236-42.

Meikle PJ, Hopwood JJ, Clague AE, et al. 1999. Prevalence of lysosomal storage disorders. JAMA, 281:249-54.

Mignani R, Feriozzi S, Pisani A, et al. 2008. Agalsidase therapy in patients with Fabry disease on renal replacement therapy: a nationwide study in Italy. Nephrol Dial Transplant, 23:1628-35.

Mignani R, Panichi V, Giudicissi A, et al. 2004. Enzyme replacement therapy with agalsidase beta in kidney transplant patients with Fabry disease: a pilot study. Kidney Int, 65:1381-5.

Nakao S, Kodama C, Takenaka T, et al. 2003. Fabry disease: Detection of undiagnosed hemodialysis patients and identification of a "renal variant" phenotype. Kidney Int, 64:801-7.

Nakao S, Takenaka T, Maeda M, et al. 1995. An atypical variant of Fabry's disease in men with left ventricular hypertrophy. $N$ Engl $J$ Med, 333:288-93. 
Ohashi T, Iizuka S, Ida H, et al. 2008. Reduced alpha-Gal A enzyme activity in Fabry fibroblast cells and Fabry mice tissues induced by serum from antibody positive patients with Fabry disease. Mol Genet Metab, 94:313-8.

Ojo A, Meier-Kriesche HU, Friedman G, et al. 2000. Excellent outcome of renal transplantation in patients with Fabry's disease. Transplantation, 69:2337-9.

Oliveira JP. 2007. Staging of Fabry disease using renal biopsies. Clin Therap, 29:S15-S6.

Oliveira JP, Ferreira S, Barceló J, et al. 2008. Effect of single nucleotide polymorphisms of the 5 ' untranslated region of the human $\alpha$-galactosidase enzyme on enzyme activity, and their frequencies in Portuguese Caucasians. J Inherit Metab Dis, in press.

ONTARGET. 2008. Telmisartan, ramipril, or both in patients at high risk for vascular events. $N$ Engl J Med, 358:1547-59.

Ortiz A, Oliveira JP, Waldek S, et al. 2008. Nephropathy in males and females with Fabry disease: cross-sectional description of patients before treatment with enzyme replacement therapy. Nephrol Dial Transplant, 23:1600-7.

Ortiz A, Oliveira JP, Wanner C, et al. 2008. Recommendations and guidelines for the diagnosis and treatment of Fabry nephropathy in adults. Nat Clin Pract Nephrol, 4:327-36.

Pastores GM, Boyd E, Crandall K, et al. 2007. Safety and pharmacokinetics of agalsidase alfa in patients with Fabry disease and end-stage renal disease. Nephrol Dial Transplant, 22:1920-5.

Pisani A, Spinelli L, Sabbatini M, et al. 2005. Enzyme replacement therapy in fabry disease patients undergoing dialysis: effects on quality of life and organ involvement. Am J Kidney Dis, 46:120-7.

Ramaswami U, Whybra C, Parini R, et al. 2006. Clinical manifestations of Fabry disease in children: data from the Fabry Outcome Survey. Acta Paediatr, 95:86-92.

Remuzzi G, Benigni A and Remuzzi A. 2006. Mechanisms of progression and regression of renal lesions of chronic nephropathies and diabetes. J Clin Invest, 116:288-96.

Ries M, Clarke JT, Whybra C, et al. 2006. Enzyme-replacement therapy with agalsidase alfa in children with Fabry disease. Pediatrics, 118:924-32.

Ries M, Ramaswami U, Parini R, et al. 2003. The early clinical phenotype of Fabry disease: a study on 35 European children and adolescents. Eur J Pediatr, 162:767-72.

Rodriguez-Iturbe B, Herrera J, Garcia R. 1985. Response to acute protein load in kidney donors and in apparently normal postacute glomerulonephritis patients: evidence for glomerular hyperfiltration. Lancet, 2:461-4.

Rosenthal D, Lien YH, Lager D, et al. 2004. A novel alpha-galactosidase a mutant (M42L) identified in a renal variant of Fabry disease. $\mathrm{Am} \mathrm{J}$ Kidney Dis, 44:e85-9.

Sakuraba H, Murata-Ohsawa M, Kawashima I, et al. 2006. Comparison of the effects of agalsidase alfa and agalsidase beta on cultured human Fabry fibroblasts and Fabry mice. J Hum Genet, 51:180-8.

Sawada K, Mizoguchi K, Hishida A, et al. 1996. Point mutation in the alphagalactosidase A gene of atypical Fabry disease with only nephropathy. Clin Nephrol, 45:289-94.

Schiffmann R. 2007. Enzyme replacement in Fabry disease: the essence is in the kidney. Ann Intern Med, 146:142-4.

Schiffmann R, Askari H, Timmons M, et al. 2007. Weekly enzyme replacement therapy may slow decline of renal function in Fabry patients who are on long-term biweekly dosing. J Am Soc Nephrol, 18:1576-83.

Schiffmann R, Kopp JB, Austin HA 3rd, et al. 2001. Enzyme replacement therapy in Fabry disease: a randomized controlled trial. JAMA, 285:2743-9.

Schiffmann R, Rapkiewicz A, Abu-Asab M, et al. 2005. Pathological findings in a patient with Fabry disease who died after 2.5 years of enzyme replacement. Virchows Arch, 448:337-43.

Schiffmann R, Ries M, Timmons M, et al. 2006. Long-term therapy with agalsidase alfa for Fabry disease: safety and effects on renal function in a home infusion setting. Nephrol Dial Transplant, 21:345-54.
Schwarting A, Dehout F, Feriozzi S, et al. 2006. Enzyme replacement therapy and renal function in 201 patients with Fabry disease. Clin Nephrol, 66:77-84.

Sessa A, Toson A, Nebuloni M, et al. 2002. Renal ultrastructural findings in Anderson-Fabry disease. $J$ Nephrol, 15:109-12.

Shen JS, Meng XL, Moore DF, et al. 2008. Globotriaosylceramide induces oxidative stress and up-regulates cell adhesion molecule expression in Fabry disease endothelial cells. Mol Genet Metab.

Sheth KJ, Roth DA, Adams MB. 1983. Early renal failure in Fabry's disease. Am J Kidney Dis, 2:651-4.

Spada M, Pagliardini S, Yasuda M, et al. 2006. High incidence of lateronset fabry disease revealed by newborn screening. Am J Hum Genet, 79:31-40.

Tahir H, Jackson LL, Warnock DG. 2007. Antiproteinuric therapy and Fabry nephropathy: Sustained reduction in proteinuria in patients receiving enzyme replacement therapy with agalsidase-beta. J Am Soc Nephrol, 18:2609-17.

Takenaka T, Teraguchi H, Yoshida A, et al. 2008. Terminal stage cardiac findings in patients with cardiac Fabry disease: An electrocardiographic, echocardiographic, and autopsy study. J Cardiol, 51:50-9.

Thurberg BL, Rennke H, Colvin RB, et al. 2002. Globotriaosylceramide accumulation in the Fabry kidney is cleared from multiple cell types after enzyme replacement therapy. Kidney Int, 62:1933-46.

Tondel C, Bostad L, Hirth A, et al. 2008. Renal biopsy findings in children and adolescents with Fabry disease and minimal albuminuria. $\mathrm{Am} \mathrm{J}$ Kidney Dis, 51:767-76.

Torra R, Algaba F, Ars E, et al. 2008. Preservation of renal function in a patient with Fabry nephropathy on enzyme replacement therapy. Clin Nephrol, 69:445-9.

Valbuena C, Carvalho E, Bustorff M, et al. 2008. Kidney biospy findings in heterozygous Fabry disease females with early nephropathy. Virchows Arch, in press

Vedder AC, Breunig F, Donker-Koopman WE, et al. 2008. Treatment of Fabry disease with different dosing regimens of agalsidase: Effects on antibody formation and GL-3. Mol Genet Metab, 94:319-25.

Vedder AC, Linthorst GE, van Breemen MJ, et al. 2007. The Dutch Fabry cohort: diversity of clinical manifestations and Gb3 levels. $J$ Inherit Metab Dis, 30:68-78.

von Scheidt W, Eng CM, Fitzmaurice TF, et al. 1991. An atypical variant of Fabry's disease with manifestations confined to the myocardium. NEngl J Med, 324:395-9.

Wang J, Lozier J, Johnson G, et al. 2008. Neutralizing antibodies to therapeutic enzymes: considerations for testing, prevention and treatment. Nat Biotechnol, 26:901-8.

Warnock DG. 2005. Fabry disease: diagnosis and management, with emphasis on the renal manifestations. Curr Opin Nephrol Hypertens, 14:87-95.

Warnock DG. 2007. Enzyme replacement therapy and Fabry kidney disease: Quo Vadis? J Am Soc Nephrol, 18:1368-70.

Whitfield PD, Calvin J, Hogg S, et al. 2005. Monitoring enzyme replacement therapy in Fabry disease - role of urine globotriaosylceramide. J Inherit Metab Dis, 28:21-33.

Whybra C, Kampmann C, Willers I, et al. 2001. Anderson-Fabry disease: clinical manifestations of disease in female heterozygotes. $J$ Inherit Metab Dis, 24:715-24.

Wilcox WR, Banikazemi M, Guffon N, et al. 2004. Long-term safety and efficacy of enzyme replacement therapy for Fabry disease. Am J Hum Genet, 75:65-74.

Wilcox WR, Oliveira JP, Hopkin RJ, et al. 2008. Females with Fabry disease frequently have major organ involvement: lessons from the Fabry Registry. Mol Genet Metab, 93:112-28.

Wraith JE, Tylki-Szymanska A, Guffon N, et al. 2008. Safety and efficacy of enzyme replacement therapy with agalsidase beta: an international, open-label study in pediatric patients with Fabry disease. $J$ Pediatr, 152:563-70. 
